Pacific Journal of Mathematics

A BIGGER BRAVER GROUP 


\title{
A BIGGER BRAUER GROUP
}

\author{
JOSEPH L. TAYLOR
}

Lat $R$ be a commutative ring with identity. In this paper we propose an extension of the concept of central separable $R$-algebra and use this to define an associated Brauer group for $R$ which contains the classical Brauer group as a subgroup. The essential difference between our notion of central separable algebra and the classical one is that we do not require that the algebra have an identity. As a consequence, our algebras need not be finitely generated or projective as $R$-modules. Nevertheless, with equality defined using an appropriate version of Morita equivalence and tensor product providing the operation, we obtain a tractable extension of the Brauer group. If $R$ is a Henselian local ring with algebraically closed residual field, our Brauer group is trivial. If $R$ is the algebra of complex valued continuous functions on a compact Hausdorff space our Brauer group is the full integral third Čech cohomology group of the underlying space, while the classical Brauer group is just the torsion subgroup.

Models for classical central separable algebras are the algebras of the form $\operatorname{End}_{R}(M)$ where $M$ is a finitely generated projective $R$ module. The class of such algebras forms the zero element of the Brauer group for $R$. Models for our central separable algebras are algebras of the form $M \otimes_{R} N$ where $M$ and $N$ are $R$-modules and multiplication is defined through a surjective $R$-module homomorphism $\lambda: N \otimes_{R} M \rightarrow R$ by the formula $\left(m_{1} \otimes n_{1}\right) \cdot\left(m_{2} \otimes n_{2}\right)=\lambda\left(n_{1} \otimes m_{2}\right)\left(m_{1} \otimes n_{2}\right)$. We do not require that $M$ and $N$ be finitely generated or projective. If $M$ is finitely generated and projective and $N=\operatorname{Hom}_{R}(M, R)$ with $\lambda$ defined by the standard pairing between $M$ and its dual, then the algebra $M \otimes_{R} N$ is just $\operatorname{End}_{R}(M)$ and we have a classical central separable $R$-algebra.

There are several equivalent definitions of separable $R$-algebra in the classical situation. For example, $A$ is separable if the multiplication map $A \otimes_{R} A \rightarrow A$ has a right inverse as an $A$-bimodule homomorphism. Equivalently, $A$ is separable if it is a projective $A$ bimodule (cf. [2], [8]). Here it is assumed that $A$ has an identity. If we drop the identity requirement, then the various conditions for separability are no longer equivalent as they stand. However, with some strengthening of hypotheses to avoid trivialities we obtain several equivalent and acceptable conditions for separability. For example, the definition we adopt in $\S 2$ is that an $R$-algebra $A$ is 
separable if it is projective as an $A$-bimodule, $A^{2}=A$, and $M A \neq A$ for each maximal ideal $M$ of $R$. Note that if $A$ has an identity then the last two conditions are redundant and $A$ is a classical separable $R$-algebra.

The first three sections of the paper are devoted to developing elementary properties of separable and (in §3) central separable $R$ algebras. Most of the results have analogues in the classical theory which are relatively trivial. The techniques required here are less trivial and quite different from the classical situation due to the lack of an identity and the absences of $R$-projectivity and finite dimensionality. The key result in $\S 3$ is Proposition 3.8 which gives a characterization of central separable $R$-algebras that is extensively used in the succeeding sections.

In $\S 4$ we discuss Morita equivalence, define an extended Brauer group, and establish its triviality for Henselian local rings with algebraically closed residual fields.

In $\S 5$ we discuss automorphisms of central separable algebras. We develop a complete analogue of the exact sequence of RosenbergZelinsky [20] relating such automorphisms to the Picard group of $R$.

Serre proved that the Brauer group of $C(X)$ for $X$ a compact Hausdorff space is the torsion subgroup of $H^{3}(X, Z)$. Following work of Dixmier-Douady [9] on $C^{*}$ algebras, we prove in $\S 6$ that our extended Brauer group is all of $H^{3}(X, Z)$ when $R$ is $C(X)$. We also prove that if $X$ is a certain kind of compact subset of a Stein space and $\mathscr{O}(X)$ is the algebra of functions holomorphic in a neighborhood of $X$, then the extended Brauer group of $\mathscr{O}(X)$ is naturally embedded as a subgroup of $H^{3}(X, Z)$. When we began writing this paper we thought we knew how to prove that this embedding was surjective. Unfortunately we discovered a gaping hole in our argument and, as a result, this question remains unsettled.

Our interest in this subject stems from an attempt to characterize the third Čech cohomology group of the maximal ideal space $\Delta_{R}$ of a commutative Banach algebra $R$. There are nice characterizations of the lower order Čech groups of $\Delta_{R}$ (e.g., $H^{2}\left(\Delta_{R}, Z\right) \cong \operatorname{Pic}(R)$ ), and it has been clear for some time that $H^{3}\left(\Delta_{R}, Z\right)$ should be identifiable with some sort of Brauer group for $R$ (cf. [7], [25]). In a joint paper with Craw and Raeburn [6] we introduced a class of Banach algebras over $R$ which can probably be used to construct such a Brauer group. This class is defined and studied using a completed topological tensor product and, as a result, it only makes sense for ground rings $R$ which are Banach or topological algebras. We had hoped that the extended Brauer group defined here would yield a strictly algebraically defined functor, defined for all rings $R$, which would yield the third Čech cohomology of $\Delta_{R}$ when $R$ is a 
Banach algebra. Unfortunately, the surjectivity question mentioned above when $R=\mathscr{O}(X)$ remains an obstacle in the way of completing this project. It may be that a purely algebraically defined Brauer group will not suffice to characterize $H^{3}\left(\Delta_{R}, Z\right)$ in all cases. To obtain such a characterization, it may be necessary to reformulate the results of this paper in the context of Banach algebras, using completed tensor products. This question is discussed in more detail in $\S 7$.

1. Splitting maps. Let $R$ be a commutative ring with identity and let $A$ be an $R$-algebra (generally without an identity).

Definition 1.1. A splitting map for $A$ is an $A$-bimodule homomorphism $\varphi: A \rightarrow A \otimes_{R} A$ which is a right inverse for the multiplication map $\pi: A \otimes_{R} A \rightarrow A$.

In the case of algebras with identity, the existence of a splitting map is equivalent to separability ([8], Prop. 1.1). In the case of algebras $A$ without identity certain degeneracies are possible even when $A$ has a splitting map. These degeneracies are undesirable. To eliminate them requires a slightly stronger definition of separability. We shall come to this in $\S 2$. In the mean time we prove what we can about algebras with splitting maps.

Another problem with algebras without identity is that the module action map $A \otimes_{A} M \rightarrow M$ ( $M$ a left $A$-module) may fail to be an isomorphism. It is trivially an isomorphism, with inverse $m \rightarrow$ $1 \otimes m: M \rightarrow A \otimes_{A} M$, if $A$ has an identity. Here, by an $A$-module we mean an $R$-module with a compatible action of the $R$-algebra $A$.

Definition 1.2. A left $A$-module will be called regular if $A \otimes_{A}$ $M \rightarrow M$ is an isomorphism. Regular right and 2-sided $A$-modules are defined analogously.

Proposition 1.1. If $A$ has a splitting map, then $A$ is a regular left (right, 2-sided) A-module.

Proof. The existence of a splitting map $\varphi$ shows that $\pi: A \otimes_{R}$ $A \rightarrow A$ is surjective. To prove that $A \otimes_{A} A \rightarrow A$ is an isomorphism we must show that $\operatorname{ker} \pi=\operatorname{im} \lambda$, where $\lambda: A \otimes_{R} A \otimes_{R} A \rightarrow A \otimes_{R} A$ is defined by $\lambda(a \otimes b \otimes c)=a \otimes b c-a b \otimes c$. However the identity $\varphi \circ \pi+\lambda \circ(1 \otimes \varphi)=1$ does this for us immediately. This proves that $A$ is regular as a left and as a right $A$-module, and this is what we mean when we say it is a regular 2 -sided $A$-module.

In what follows, the term "relative" will always refer to the 
ground ring $R$. For example, $P$ is relatively projective means that homomorphisms of $P$ into a quotient module lift provided the quotient map is $R$-split.

Proposition 1.2. If $A$ has a splitting map $\varphi$ and $M$ is a regular left $A$-module with action map $\pi_{M}: A \otimes_{R} M \rightarrow M$, then $\varphi$ induces a left $A$-module homomorphism $\varphi_{M}: M \rightarrow A \bigotimes_{R} M$ which is a right inverse for $\pi_{M}$. The analogous statement holds for right $A$-modules.

Proof. If we let $\nu$ denote the isomorphism $M \rightarrow A \otimes_{A} M$, then $\varphi_{M}$ is just the composition of the three maps

$$
M \stackrel{\nu}{\longrightarrow} A \otimes_{A} M \stackrel{\varphi \otimes 1}{\longrightarrow} A \otimes_{R} A \otimes_{A} M \stackrel{1 \otimes_{\nu}^{-1}}{\longrightarrow} A \otimes_{R} M .
$$

Proposition 1.3. If $A$ has a splitting map then every regular left (right) A-module is relatively projective.

Proof. Let $M$ be a regular left $A$-module. Suppose $\mu: L \rightarrow N$ is a surjective left $A$-module homomorphism which is $R$-split by $\nu: N \rightarrow$ $L$ and suppose $\alpha: M \rightarrow N$ is a left $A$-module homomorphism. Then we have the commutative diagram

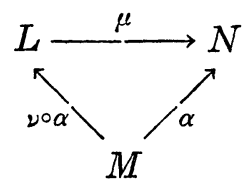

with $\mu$ and $\alpha A$-module maps and $\nu \circ \alpha$ an $R$-module map. Applying $A \otimes_{R}(\quad)$ yields a commutative diagram of $A$-module homomorphisms

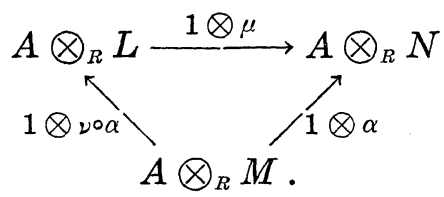

If each of $1 \otimes \nu \circ \alpha$ and $1 \otimes \alpha$ is preceded by the map $\varphi_{M}: M \rightarrow A \otimes_{R} M$ of Proposition 1.2 and followed by the appropriate action map, we obtain the commutative diagram

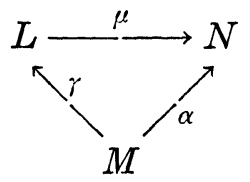

with $\gamma=\pi_{L^{\circ}}(1 \otimes \nu \circ \alpha) \circ \varphi_{M}$ the required $A$-module lifting of $\alpha$. Thus, $M$ is relatively projective. 
CoRollary. If $A$ has a splitting map, then $A$ is a relatively projective left (right, 2-sided) A-module.

Note that the argument in Proposition 1.3 is just a little bit different from the standard argument for the analogous fact for algebras with identity. Also, in the case of algebras with identity, a trivial argument gives that relatively projective modules are relatively flat. We can prove an analogous result but the argument requires a certain twist.

Proposition 1.4. If $A$ has a splitting map, then every regular A-module is relatively flat.

Proof. We first prove that $A$ is relatively flat as a right $A$ module. If $M$ is a left $A$-module, consider the sequence

$$
A \otimes_{R} A \otimes_{R} M \stackrel{\lambda}{\longrightarrow} A \otimes_{R} M \stackrel{\mu}{\longrightarrow} A \otimes_{A} M \longrightarrow 0
$$

where $\lambda(a \otimes b \otimes m)=a \otimes b m-a b \otimes m$ and $\mu$ is, by definition, the quotient map modulo the image of $\lambda$. If $A$ has a splitting map $\varphi$, then $-\lambda \circ(\varphi \otimes 1)$ is a projection onto the image of $\lambda$. This projection is natural in the sense that it commutes with the induced map $A \otimes_{R} M \rightarrow A \otimes_{R} N$ whenever $M \rightarrow N$ is an $A$-module homomorphism. In other words, $\mu: A \otimes_{R} M \rightarrow A \otimes_{A} M$ is naturally split as a left $A$ module homomorphism. It follows that if $0 \rightarrow L \rightarrow M \rightarrow N \rightarrow 0$ is an exact sequence of left $A$-modules for which exactness is preserved by $A \otimes_{R}()$, then exactness is also preserved by $A \otimes_{A}($ ). In particular, $A \otimes_{A}()$ will preserve the exactness of $R$-split exact sequences, i.e., $A$ is relatively flat as a right $A$-module.

Now if $P$ is any regular right $A$-module then $P$ is a direct summand of $P \otimes_{R} A$ by Proposition 1.2. That $A$ is relatively flat implies the same is true of $P \otimes_{R} A$ and $P$.

This leads to the following result on the stability of regularity:

Proposition 1.5. If $0 \rightarrow L \rightarrow M \rightarrow N \rightarrow 0$ is an $R$-split exact sequence of left (right) $A$-modules and $A$ has a splitting map, then the regularity of any two of $L, M, N$ implies the regularity of the third.

Proof. Suppose $L, M, N$ are left $A$-modules. By Proposition 1.4, $A$ is a relatively flat right $A$-module. Hence, we have the commutative diagram 


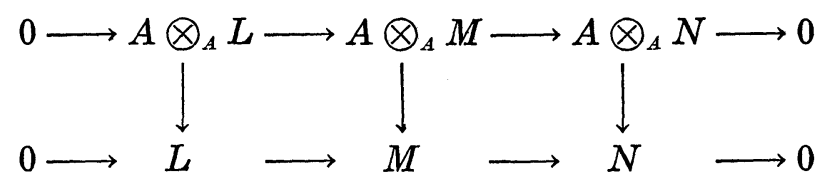

with both rows exact. Thus, if any two verticle maps are isomorphisms, so is the third.

Proposition 1.6. If $A$ has a splitting map, $M$ is a regular left (right) $A$-module, and $L \subset M$ is a submodule with $A L=L$, then $L$ is regular.

Proof. Consider the diagram in the proof of Proposition 1.5 with $N=M / L$. Since $A \otimes_{A} M \rightarrow M$ is an isomorphism, $A \otimes_{A} L \rightarrow L$, is automatically injective; since $A \cdot L=L$, it is also surjective.

Proposition 1.7. Let A have a splitting map. If $M$ is a regular left (right) $A$-module and $L \subset M$ a submodule with $A L=L$, then if $L$ is an $R$-module direct summand of $M$ it is also an A-module direct summand of $M$.

Proof. Combine Proposition 1.3 and Proposition 1.6.

The following proposition will allow us to apply Proposition 1.7 to 2-sided $A$-modules also:

Proposition 1.8. If $A$ has a splitting map, so does the algebra $A^{e}=A \otimes_{R} A^{o p}$.

Proof. If $\varnothing$ is a splitting map for $A$ then $\phi \otimes S \circ \varphi$ is a splitting map for $A^{e}$, where $T(a \otimes b)=b \otimes a$.

Now regarding an $A$-bimodule as a left $A^{e}$-module allows us to conclude that Proposition 1.7 is true with left modules replaced by 2-sided modules. If we apply this to the case where $M=A$ and $L=I$ is a 2 -sided ideal of $A$, we get:

Proposition 1.9. Let $A$ have a splitting map and suppose $I$ is a 2-sided ideal of $A$ with $A I A=I$. Then if $I$ is an $R$-module direct summand it is also an A-bimodule direct summand.

2. Separable algebras. We are now prepared to define separable $R$-algebras. At first glance the definition (Def. 2.1 below) appears much stronger than the simple existence of a splitting map. However, eventually we shall show they are practically the same thing. 
If $A$ is an $R$-algebra we will denote $\operatorname{End}_{A^{e}}(A)$ by $Z(A)$. If $A$ had an identity this would be the center of $A$. In the absence of an identity, there is no natural way to regard $Z(A)$ as a subalgebra of $A$. However, at least we have:

Proposition 2.1. If $A^{2}=A$ then $Z(A)$ is a commutative $R$ algebra.

Proof. If $\alpha, \beta \in Z(A)$ and $a, b \in A$ then $\alpha \circ \beta(a b)=\alpha(a \beta(b))=$ $\alpha(a) \beta(b)=\beta(\alpha(a) b)=\beta \circ \alpha(a b)$. Hence, $Z(A)$ is commutative if $A^{2}=A$,

Definition 2.1. Let $A$ be an $R$-algebra. We shall call $A$ separable provided:

(a) $A^{2}=A$;

(b) $A$ is a projective $A^{e}$-module; and

(c) for each maximal ideal $M \subset Z(A), M A \neq A$.

Condition (c) is a nondegeneracy condition. It implies, in particular, that $A \neq(0)$.

Proposition 2.2. If $A$ is separable then $A$ has a splitting $\operatorname{map}$.

Proof. Since $\pi: A \otimes_{R} A \rightarrow A$ is surjective by (a) and $A$ is $A^{e}$ projective by (b), $\pi$ has a right inverse $\varphi$ as an $A^{e}$-module homomorphism. Such a thing is a splitting map.

Definition 2.2. We shall call $A$ central if the natural map $R \rightarrow Z(A)$ is an isomorphism. Observe this makes $A$ faithful.

To avoid confusion in what follows, the multiplication in $A^{e}$ will be indicated through the use of a dot between the factors, as will the left module action of $A^{e}$ on $A$. We shall not employ the $\operatorname{dot}$ when multiplying elements of $A$ or writing the left or right $A$ module action of $A$ on $A \otimes A$.

Proposition 2.3. Let $A$ be a nonzero central $R$-algebra with a splitting map $\varphi$. If $R$ is a field, then $\varphi(A) \cdot A^{e}=A^{e}$ and $A^{e}$ is also central.

Proof. The space $\varphi(A) \cdot A^{e}$ is two-sided ideal of $A^{e}$. We first show that it cannot be the zero ideal.

Let $N=\{n \in A$; $a n=n b=0$ for all $a, b \in A\}$ and initially assume 
that $N=(0)$. Then the family of linear functions from $A$ to $R$ of the form $a \rightarrow f(a b)$ with $b \in A$ and $f$ in the dual of $A$, is a separating family of linear functionals on the $R$-vector space $A$. The same is true of the family of functionals of the form $a \rightarrow g(c a)$ for $c \in A$ and $g$ in the dual of $A$. It follows that tensor products of functionals of these two forms yield a separating family of linear functionals on $A \otimes_{R} A$. Thus, if $\alpha \in A^{e}$ and $\alpha \cdot(b \otimes c)=0$ for all $b, c \in A$, then $\alpha=0$.

Returning to the case where $N$ may not be zero, if we apply the above argument to $A / N$ we conclude that $\alpha \cdot A^{e}=0$ implies that $\alpha \in A \otimes_{R} N+N \otimes_{R} A$. This, in turn, implies $\pi \alpha=0$ where $\pi: A \otimes_{R} A \rightarrow A$ is the multiplication map. Since $\pi \circ \varphi=1$ we conclude that $\varphi(A) \cdot A^{e} \neq(0)$.

Since $A^{e} \cdot \varphi(A)=\varphi\left(A^{e} \cdot A\right)=\varphi(A)$ we have that $\varphi(A) \cdot A^{e}$ is the two-sided ideal in $A^{e}$ generated by $\varphi(A)$. Hence, it is an $\left(A^{e}\right)^{e}$ module direct summand of $A^{e}$ by Proposition 1.9. This means there is a projection $p \in Z\left(A^{e}\right)$ with $p A^{e}=\varphi(A): A^{e}$. Clearly, $p$ is not multiplication by an element of $R$ (since $R$ is a field) unless $p=1$ and $\varphi(A) \cdot A^{e}=A^{e}$. Thus, the proof will be finished if we can show that $A^{e}$ is central.

Let $\alpha \in Z\left(A^{e}\right)$ be given. Then for $a \in A$ and $f$ in the dual of $A$, the map $b \rightarrow(f \otimes 1) \alpha(a \otimes b)$ belongs to $Z(A)$ and, hence, is given by an element $r(a \cdot f) \in R$. Then for any functional $g$ in the dual of $A$ we have:

$$
(f \otimes g) \alpha(a \otimes b)=r(a, f) g(b) .
$$

Similarly, $a \rightarrow(1 \otimes g) \alpha(a \otimes b)$ is given by an element $s(b, g) \in R$. Then

$$
(f \otimes g) \alpha(a \otimes b)=s(b, g) f(a) .
$$

Thus, $s(b, g) f(a)=r(a, f) g(b)$, which implies that there exists $k \in R$ such that $s(b, g)=k g(b)$ and $r(a, f)=k f(a)$. Then

$$
(f \otimes g) \alpha(a \otimes b)=k(f \otimes g)(a \otimes b)
$$

and we conclude that $\alpha$ is given by multiplication by $k \in R$. Thus, $A^{e}$ is central.

Proposition 2.4. Let $A$ be separable and let $I \subset A$ be a twosided ideal. Then every element of $Z(A / I)$ lifts to an element of $Z(A)$.

Proof. If $\alpha \in Z(A / I)$ we wish to find $\beta \in Z(A)$ such that the diagram 


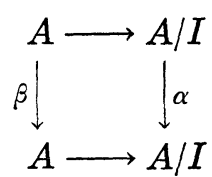

is commutative. However, the existence of $\beta$ follows immediately from the fact that $A$ is a projective $A^{e}$-module and the identities $Z(A)=\operatorname{End}_{A^{e}}(A), Z(A / I)=\operatorname{End}_{A^{e}}(A / I)$.

If $A$ is an algebra with a splitting map $\varnothing$ and $b \in A$, then $a \rightarrow$ $\varphi(a) \cdot b: A \rightarrow A$ defines an element of $Z(A)$. If we denote this element by $\mu_{\varphi}(b)$, then $\mu_{\varphi}: A \rightarrow Z(A)$ is an $R$-module homomorphism. In fact, it is a $Z(A)$-module homomorphism since if $\beta \in Z(A)$ then $\beta \mu_{\varphi}(b) a=$ $\beta(\varphi(a) \cdot b)=\varphi(a) \cdot \beta(b)=\mu_{\varphi}(\beta(b) a)$ for all $a, b \in A$.

Proposition 2.5. If $A$ is separable and $\varphi$ is a splitting map for $A$, then $\mu_{\varphi}: A \rightarrow Z(A)$ is surjective. Furthemore, if $b \in A$ is an element for which $\mu_{\varphi}(b)=1$, then $Z(A) b$ is a $Z(A)$-module direct summand of $A$ which is isomorphic to $Z(A)$.

Proof. If $\mu_{\varphi}$ is not surjective, then its image is a proper twosided ideal of $Z(A)$ and, hence, is contained in a maximal ideal $M$. Now by (c) of Definition 2.1, $B=A / M A \neq(0)$. Furthermore, $Z(B)$ is the field $Z(A) / M$ by Proposition 2.4.

Note that $\varphi$ induces a splitting map $B \rightarrow B \otimes_{R} B$ for $B$, which when composed with $B \otimes_{R} B \rightarrow B \otimes_{Z(B)} B$ yields a splitting map $\tilde{\Phi}$ for $B$ regarded as a $Z(B)$-algebra. Hence, Proposition 2.3 applies and we conclude that $\widetilde{\varphi}(B) \cdot B^{e}=B^{e}$. On applying the multiplication map $B^{e} \rightarrow B$ this yields $\widetilde{\varphi}(B) \cdot B=B$. On the other hand, by the definition of $\mu_{\varphi}$ and $M$, we have $\varphi(A) \cdot A \subset M A$, which implies $\widetilde{\varphi}(B) \cdot B=(0)$. The resulting contradiction shows that $\mu_{\varphi}$ must be surjective.

Proposition 2.6. If $A$ is an R-algebra, then $A$ is separable if and only if $A$ has a splitting map $\varphi$ for which $\mu_{\varphi}$ is surjective.

Proof. In view of Proposition 2.5, we need only show that if $A$ has a splitting map $\varnothing$ with $\mu_{\varphi}$ surjective then $A$ is separable.

The existence of $\phi$ immediately gives $A^{2}=A$. Suppose $\alpha: M \rightarrow$ $N$ and $\beta: A \rightarrow N$ are $A^{e}$-module homomorphisms and $\alpha$ is surjective. If $\mu_{\varphi}$ is surjective we choose $a_{0} \in A$ with $\mu_{\varphi}\left(a_{0}\right)=1$ and $m_{0} \in M$ with $\alpha\left(m_{0}\right)=\beta\left(a_{0}\right)$. Then $\gamma(a)=\varphi(a) \cdot m_{0}$ defines a lifting $\gamma: A \rightarrow M$ of $\beta$. In fact,

$$
\begin{aligned}
\alpha \circ \gamma(a) & =\alpha\left(\varphi(a) \cdot m_{0}\right)=\varphi(a) \cdot \alpha\left(m_{0}\right)=\varphi(a) \cdot \beta\left(a_{0}\right) \\
& =\beta\left(\varphi(a) \cdot a_{0}\right)=\beta\left(\mu_{\varphi}\left(a_{0}\right) a\right)=\beta(a) .
\end{aligned}
$$


Hence, $A$ is a projective $A^{e}$-module.

Finally, the map $b \rightarrow \mu_{\varphi}(b) a_{0}$, where $\mu_{\varphi}\left(a_{0}\right)=1$, expresses $Z(A) a_{0}$ as a $Z(A)$-module direct summand of $A$ which is isomorphic to $Z(A)$. Thus, we cannot have $M A=A$ for a proper ideal $M \subset Z(A)$. This completes the proof that $A$ is separable.

Proposition 2.7. Let $A$ and $B$ be $R$-algebras and let $I$ be a regular two-sided ideal of $A$; then

(a) if $A$ is separable, so is $A / I$;

(b) if $A$ is central separable, then $A / I$ is central over $R / \mu_{\varphi}(I)$;

(c) if $A$ and $B$ are separable, so is $A \otimes_{R} B$; and

(d) if $A$ and $B$ are central separable, so is $A \otimes_{R} B$.

Proof. If $\phi$ is a splitting map for $A$, then the regularity of $I$ implies that $\varphi$ maps $I$ into $I \otimes_{R} A$ and, hence, that $\varphi$ induces a well defined splitting map $\widetilde{\Phi}$ for $A / I$. Clearly, if $\mu_{\varphi}$ has 1 in its image so does $\mu_{\tilde{\varphi}}$. Thus, $A / I$ is separable. That $A / I$ is $R / \mu_{\varphi}(I)$ central if $A$ is $A$-central follows from Proposition 2.4.

Now suppose $A$ and $B$ are separable with splitting maps $\phi$ and $\psi$ with $\mu_{\varphi}$ and $\mu_{\psi}$ surjective. Then clearly $\phi \otimes \psi$ is a splitting map for $A \otimes_{R} B$ and $\mu_{\varphi \otimes \psi}(a \otimes b)=\mu_{\varphi}(a) \mu_{\psi}(b)$-which implies that $\mu_{\varphi \otimes \psi}$ is surjective and $A \otimes_{R} B$ is separable. If $A$ and $B$ are also central, then $\mu_{\varphi}$ and $\mu_{\psi}$ are $R$-valued. It follows that $\mu_{\varphi \otimes \psi}$ is $R$-valued as well and, hence, that $A \otimes_{R} B$ is central.

3. Central separable algebras. In this section we establish analogues of several of the basic results from the classical theory of central separable algebras with identity. This material lays the groundwork for the definition of the Brauer group in $\S 4$.

Let $A$ be a central separable algebra over $R$ and let $\varphi$ be a splitting map for $A$. Recall the map $\mu_{\varphi}: A \rightarrow R$ of Proposition 2.5, which is a surjective $R$-module homomorphism characterized by the identity $\mu_{\varphi}(b) a=\varphi(a) \cdot b$. If $J$ is a two-sided ideal of $A$, then $\mu_{\varphi}(J)$ is an ideal of $R$ and the above identity shows that $\mu_{\varphi}(J) A \subset J$. The following is an analogue of Lemma 3.5, Chapter 2 of [8].

Proposition 3.1. With $A$ and $\varphi$ as above, if $J$ is a maximal two-sided ideal, then $\mu_{\varphi}(J)$ is a maximal ideal of $R$ and $\mu_{\varphi}(J) A=$ $A J A$.

Proof. If $M$ is an ideal of $R$ such that $\mu_{\varphi}(J) \subset M$, then $J+M A$ is a two-sided ideal of $A$ containing $J$. Since $\mu_{\phi}(J+M A) \subset M, J+$ $M A$ is a proper ideal. The maximality of $J$ implies $M A \subset J$ and, hence, $M=\mu_{\varphi}(M A) \subset \mu_{\varphi}(J)$. We conclude that $\mu_{\varphi}(J)$ is maximal. 
Now $A / \mu_{\varphi}(J) A$ is a central separable algebra (Prop. 2.7) over the field $R / \mu_{\varphi}(J)$. It follows from Proposition 1.9 that $A / \mu_{\varphi}(J) A$ can have no proper nonzero regular ideals. Hence, if $\widetilde{J}$ is the image of $J$ in $A / \mu_{\varphi}(J) A$, then $A \widetilde{J} A=(0)$. We conclude that $A J A \subset \mu_{\varphi}(J) A$. Since $\mu_{\varphi}(J) A \subset J$ and $A^{2}=A$, it follows that $A J A=\mu_{\varphi}(J) A$, as claimed.

The next proposition would be trivial if we were working with algebras with identity:

Proposition 3.2. If $A$ is central separable, then every two-sided ideal of $A$ is contained in a maximal two-sided ideal.

Proof. If $J \subset A$ is a two-sided ideal, then $\mu_{\varphi}(J)$ is an ideal of $R$ with $\mu_{\varphi}(J) A \subset J$. If $M$ is a maximal ideal of $R$ containing $\mu_{\varphi}(J)$ and $K$ is any proper two-sided ideal of $A$ containing $M A$, then $\mu_{\varphi}(K)=M$ and, necessarily $A K A=M A$. Thus, although $M A$ may not be maximal, the ideal $I_{M}=\{a \in A: b a c \in M A$ for all $b, c \in A\}$ is maximal. Since $I_{M}$ contains $M A$ it also contains $J$.

Proposition 3.3. If $A$ is central separable, then $\varphi(A) \cdot A^{e}=A^{e}$.

Proof. If $\varphi(A) \cdot A^{e}$ is not all of $A^{e}$ then it is a proper regular two-sided ideal in $A^{e}$. By Proposition 2.7, $A^{e}$ is a central separable algebra. By Proposition 3.2, $\varphi(A) \cdot A^{e}$ is contained in a maximal ideal $J$, we have $A^{e} \cdot J \cdot A^{e}=M A^{e}$ for some maximal ideal $M$ of $R$ by Proposition 3.1. Since $\varphi(A) \cdot A^{e}$ is regular and contained in $J$, it is also contained in $A^{e} \cdot J \cdot A^{e}=M A^{e}$. This is impossibe since $\pi\left(\varphi(A) \cdot A^{e}\right)=$ $A \not \subset M A$ by Proposition 2.6. We conclude that $\varphi(A) \cdot A^{e}$ is not a proper ideal of $A^{e}$.

Proposition 3.4. If $A$ is central separable, $\varphi$ and $\mu_{\varphi}$ are as above, and $N=\{a \in A: A a A=(0)\}$, then a two-sided ideal $J \subset A$ is contained in $N$ if and only if $\mu_{\varphi}(J)=(0)$.

Proof. If $J \subset N$ then $\mu_{\varphi}(J) A=\varphi(A) \cdot J=(0)$ and, hence, $\mu_{\varphi}(J)=$ (0). On the other hand, if $\mu_{\varphi}(J)=(0)$ then $A^{e} \cdot J=\varphi(A) \cdot A^{e} \cdot J \subset$ $\varphi(A) \cdot J=\mu_{\varphi}(J) \cdot A=(0)$.

We can now prove a version of the ideal correspondence (cf. [8], Ch. 2, Cor. 3.7):

Proposition 3.5. Let $A$ be central separable with $\varphi$ and $\mu_{\varphi}$ as before. Then the map $M \rightarrow M A$ is a bijection from the set of ideals of $R$ to the set of regular two-sided ideals of $A$. Furthermore, if $J$ 
is any two-sided ideal of $A$, then $A J A$ is regular and equal to $M A$ for $M=\mu_{\varphi}(J)$.

Proof. Since $\mu_{\varphi}(M A)=M$ the map $M \rightarrow M A$ is injective. If $J$ is a two-sided ideal of $A$, then $M=\mu_{\varphi}(J)$ is an ideal of $R$ and $M A$ is a regular two-sided ideal of $A$ contained in $J$. The algebra $A / M A$ is central separable over $R / M$ by Proposition 3.3. Since $M=\mu_{\varphi}(J)$, Proposition 3.4 implies that the image of $J$ in $A / M A$ is contained in the ideal $N$ for $A / M A$. Hence, $A J A \subset M A$, which implies $A J A=$ $M A$ since $M A \subset J$ and $M A$ is regular. If $J$ happens to be regular, then $J=M A$.

There are four module actions of $A$ on $A \otimes_{R} A$-the first and second left actions (referring to which factor in $A \otimes_{R} A$ is being acted upon) and the first and second right actions.

Definition 3.1. Let $\Omega$ (or $\Omega(A)$ if the $A$-dependence needs exhibiting) denote the $R$-module consisting of all $R$-module homomorphisms $\omega: A \otimes_{R} A \rightarrow A \bigotimes_{R} A$ with the property that $\omega$ is a homomorphism for the four module actions described above except that the two right actions are interchanged.

We may also regard $A \otimes_{R} A$ as an $A^{e}$-bimodule in two ways: first, by identifying it with $A^{e}$ and using the multiplication in $A^{e}$, and, second, by identifying $A \otimes_{R} A$ with $A_{l} \otimes_{R} A_{r}$ where $A_{l}$ is $A$ regarded as the left $A^{e}$-module with operation $(b \otimes c) \cdot a=b a c$ and $A_{r}$ is $A$ regarded as the right $A^{e}$-module with operation $a \cdot(b \otimes c)=$ $c a b$. If we denote the $A^{e}$-bimodule $A_{l} \otimes_{R} A_{r}$ by $A^{p}$ then $\Omega$ may be regarded as the space of $A^{e}$-bimodule homomorphisms $\omega: A^{p} \rightarrow A^{e}$ or, alternatively, as the space of $A^{e}$-bimodule homomorphisms $\omega: A^{e} \rightarrow A^{p}$.

Note that the composition of two elements of $\Omega$ may be regarded as an element of $Z\left(A^{e}\right)$. Hence, if $A^{e}$ is central then composition of two elements of $\Omega$ yields an element of $R$.

If $\omega \in \Omega$ and $b \in A$, then $a \rightarrow \pi \circ \omega(a \otimes b)$ is an $A$-bimodule endomorphism of $A$, i.e., an element of $Z(A)$. We denote this element by $\operatorname{tr}_{\omega}(b)$. Then $\pi \circ \omega(a \otimes b)=\operatorname{tr}_{\omega}(b) a$.

Proposition 3.6. If $A^{2}=A$ and $\omega \in \Omega(A)$, then $\operatorname{tr}_{\omega}$ is a $Z(A)$ module homomorphism of $A$ to $Z(A)$ and $\operatorname{tr}_{\omega}(a b)=\operatorname{tr}_{\omega}(b a)$ for all $a, b \in A$.

Proof. For $a, b, c, \in A$ we have

$$
\operatorname{tr}_{\omega}(a b) c=\pi \circ \omega(c \otimes a b)=\omega(c \otimes a) \cdot b=\pi \circ \omega(c \otimes b a)=\operatorname{tr}_{\omega}(b a) c .
$$


If $Z \in z(A)$ and $a, b, c \in A$ we have

$$
\begin{aligned}
\operatorname{tr}_{\omega}(z(a b)) c & =\pi \circ \omega(c \otimes z(a b))=\pi \circ \omega(c \otimes z(a) b) \\
& =\omega(c \otimes b) \cdot z(a)=z(\omega(c \otimes b) \cdot a)=z\left(\operatorname{tr}_{\omega}(a b)\right) .
\end{aligned}
$$

Hence, $\operatorname{tr}_{\omega}$ is a $Z(A)$-module homomorphism if $A^{2}=A$.

Suppose $A$ is central, then the pairing $\Omega \times \Omega \rightarrow Z(A)=R$, given by composition, yields an $R$-module homomorphism $\rho: \Omega \otimes_{R} \Omega \rightarrow R$.

There is a map from $A \otimes_{R} A \otimes_{R} \Omega$ to $A \otimes_{R} A$ given by $a \otimes b \otimes$ $\omega \rightarrow \omega(a \otimes b)$. This can be regarded as an $A^{e}$-bimodule map $\theta: A^{p} \otimes_{R} \Omega \rightarrow A^{e}$. In the same vein, there is a map tr: $A \otimes_{R} \Omega \rightarrow Z(A)$ defined by $\operatorname{tr}(a \otimes \omega)=\operatorname{tr}_{\omega}(a)$. If $A$ is central then tr has its values in $R$. The map $\theta$ will be called the separator for $A$ while $\operatorname{tr}$ will be called the trace.

Proposition 3.7. If $A$ is a central separable algebra then

(1) $\rho: \Omega \otimes_{R} \Omega \rightarrow R$ is an $R$-module isomorphism;

(2) $\theta: A^{p} \otimes_{R} \Omega \rightarrow A^{e}$ is an isomorphism; and

(3) $\operatorname{tr}: A \otimes_{R} \Omega \rightarrow R$ is surjective.

Proof. All three statements follow from the existence of a rich supply of elements of $\Omega$. We may construct such elements as follows: Let $\varphi$ be a splitting map for $A$ and set $\varphi^{t}=S \circ \varphi$ where $S(a \otimes b)=$ $b \otimes a$. If $\alpha \in A^{e}$ then $\alpha$ defines an element $\omega_{\alpha} \in \Omega$ by $\omega_{\alpha}(a \otimes b)=$ $\varphi(a) \cdot \alpha \cdot \varphi^{t}(b)$. Then the fact that $\theta: A \otimes_{R} A \otimes_{R} \Omega \rightarrow A \bigotimes_{R} A$ is surjective follows immediately from the identity $\varphi(A) \cdot A^{e} \cdot \varphi^{t}(A)=A^{e}$. This, in turn, follows from Proposition 3.3.

Now consider the commutative diagram

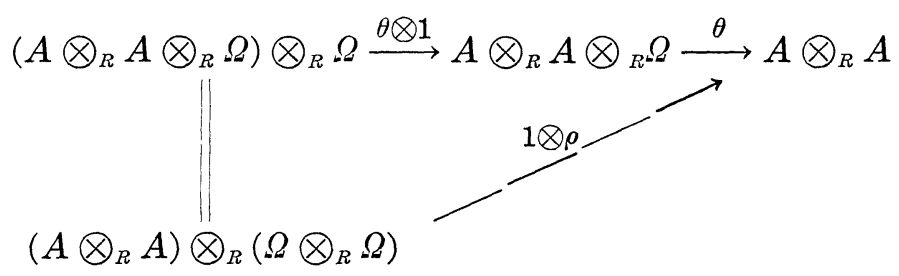

Since $\theta$ is surjective $\rho$ must be surjective, otherwise its image would be a proper ideal $M$ of $R$ with $M A^{e}=A^{e}$. This is impossible since $A^{e}$ is separable.

Since $\rho$ is surjective, we may choose $\Sigma \omega_{i} \otimes \omega_{i}^{\prime} \in \Omega \otimes_{R} \Omega$ such that $\Sigma \omega_{i} \circ \omega_{i}^{\prime}=1$. We set $\sigma(r)=r \Sigma \omega_{i} \otimes \omega_{i}^{\prime}$ for $r \in R$. Then $\sigma: R \rightarrow$ $\Omega \otimes_{R} \Omega$ is an inverse for $\rho$. In fact, $\rho \circ \sigma=1$ is clear, while

$$
\begin{aligned}
\sigma \circ \rho\left(\omega \otimes \omega^{\prime}\right) & =\left(\omega \circ \omega^{\prime}\right) \Sigma \omega_{i} \otimes \omega_{i}^{\prime}=\Sigma \omega\left(\omega^{\prime} \circ \omega_{i}\right) \otimes \omega_{i}^{\prime} \\
& =\Sigma \omega \otimes\left(\omega^{\prime} \otimes \omega_{i}\right) \omega_{i}^{\prime}=\Sigma \omega \otimes \omega^{\prime}\left(\omega_{i} \circ \omega_{i}^{\prime}\right)=\omega \otimes \omega^{\prime} .
\end{aligned}
$$


This establishes (1).

Returning to the diagram (*), note that since $\rho$ is an isomorphism, $\theta$ must be injective. Thus, $\theta$ is an isomorphism and (2) is established.

To prove (3), note that $1 \otimes \operatorname{tr}=\pi \circ \theta$. Thus, since $\pi$ and $\theta$ are surjective the image of $\operatorname{tr}$ is an ideal $M$ of $R$ with $M A=A$. Hence, $M=R$ and $\operatorname{tr}$ is surjective.

Condition (3) above turns out to be necessary and sufficient for $A$ to be separable. In fact, a somewhat stronger result is:

Proposition 3.8. Let $A$ be a faithful $R$-algebra and let $\widetilde{\Omega} \subset \Omega$ be an $R$-submodule. If $\operatorname{tr}$ maps $A \otimes_{R} \widetilde{\Omega}$ onto $R \subset Z(A)$ then $A$ is central separable and $\widetilde{\Omega}=\Omega$.

Proof. By Proposition 3.6 each $\operatorname{tr}_{\omega}$ for $\omega \in \widetilde{\Omega}$ is a $Z(A)$-module homomorphism (provided $A^{2}=A$ ). Thus tr must map $A \otimes_{R} \widetilde{\Omega}$ onto $Z(A)$, from which we conclude that $A$ is central. That $A^{2}=A$ follows from the identity $1 \otimes \operatorname{tr}=\pi \circ \theta$ and the hypothesis on $\operatorname{tr}$.

If $A$ is separable then Proposition 3.7 implies $\Omega$ is an invertible $R$-module. Then the only way $\widetilde{\Omega}$ could be a proper $R$-submodule would be if it were contained in $M \Omega$ for some proper ideal $M \subset R$. The hypothesis on trace makes this impossible.

To complete the proof we must prove that $\operatorname{tr}: A \otimes_{R} \Omega \rightarrow R$ surjective implies $A$ is separable.

From the above we have that $A^{2}=A$. If $M \subset R$ is an ideal for which $M A=A$, then $\operatorname{tr}(A \otimes \Omega)=\operatorname{tr}(M A \otimes \Omega) \subset M$. Hence, if $\operatorname{tr}$ is surjective $M=R$. Thus, conditions (a) and (c) Definition 2.1 are satisfied.

To complete the proof, we show that $A$ is projective as a left $A^{e}$-module. Since $\operatorname{tr}$ is surjective and $\pi$ is surjective, we may choose an element $\Sigma b_{i} \otimes c_{i} \otimes \omega_{i} \in A \otimes_{R} A \otimes_{R} \Omega$ such that $\Sigma \operatorname{tr}_{\omega_{i}}\left(b_{i} c_{i}\right)=1$. We define $\varphi_{i} \in \operatorname{Hom}_{A^{e}}\left(A, A^{e}\right)$ by $\varphi_{i}(a)=\omega_{i}\left(\alpha \otimes c_{i}\right)$ and note that

$$
\Sigma \varphi_{i}(a) \cdot b_{i}=\Sigma \pi \circ \omega_{i}\left(a \otimes b_{i} c_{i}\right)=\left(\Sigma \operatorname{tr}_{\omega_{i}}\left(b_{i} c_{i}\right)\right) a=a .
$$

Now suppose $\mu: M \rightarrow N$ and $\nu: A \rightarrow N$ are $A^{e}$-module homomorphisms with $\mu$ surjective. We choose $m_{i} \in M$ such that $\mu\left(m_{i}\right)=\nu\left(b_{i}\right)$ and define $\gamma: A \rightarrow M$ by $\gamma(a)=\Sigma \varphi_{i}(a) \cdot m_{i}$. Then $\mu \cdot \gamma(a)=\Sigma \phi_{i}(a) \cdot \mu\left(m_{i}\right)=$ $\Sigma \varphi_{i}(a) \cdot \nu\left(b_{i}\right)=\nu\left(\Sigma \varphi_{i}(a) \cdot b_{i}\right)=a$. Thus, $\gamma$ lifts $\nu$ and $A$ is projective.

The module $\Omega$ is rather mysterious. For central separable $A, \Omega$ is an invertible $R$-module by Proposition 3.7 and, hence, determines an element of $\operatorname{Pic}(R)$. In fact, this element is a square root of unity since $\Omega \otimes_{R} \Omega \cong R$. We conjecture that $\Omega \cong R$ for all central separable $A$. We shall see that this is true for a wide variety of algebras $A$. For example, if $A$ has an identity $e$, then $\omega \rightarrow \operatorname{tr}_{\omega}(e): \Omega \rightarrow R$ is an isomorphism. If $\omega$ is chosen so that $\operatorname{tr}_{\omega}(e)=1$, then $\omega(e \otimes e)=$ 
$\Sigma c_{i} \otimes d_{i}$ is an idempotent of $A^{e}$ with the property that $\omega(a \otimes b)=$ $\Sigma a c_{i} b \otimes d_{i}=\Sigma c_{i} \otimes b d_{i} a$.

If $\Omega$ is isomorphic to $R$ (determines the identity element of $\operatorname{Pic}(R)$ ), then the separator $\theta$ determines an isomorphism $A^{p} \rightarrow A^{e}$. This is not unique but depends on the choice of the isomorphism $\Omega \rightarrow R$. Clearly the isomorphism $A^{p} \rightarrow A^{e}$ defined by $\theta$ is unique up to an invertible multiplicative factor from $R$. Similarly, $\Omega \cong R$ implies that tr yields a trace from $A$ to $R$ which is unique up to a normalization factor from $R$.

If there exists $\omega \in \Omega$ and $b \in A$ such that $\operatorname{tr}_{\omega}(b)=1$, then $\omega^{\prime} \rightarrow$ $\operatorname{tr}_{\omega^{\prime}}(b): \Omega \rightarrow R$ is an isomorphism. Since $\operatorname{tr}: A \otimes_{R} \Omega \rightarrow R$ is surjective for a central separable algebra $A$, there are always finite sets of elements $\left\{\omega_{i}\right\}$ and $\left\{b_{i}\right\}$ so that $\Sigma \operatorname{tr}_{\omega_{i}}\left(b_{i}\right)=1$. If $R$ is a local ring, we must have $\operatorname{tr}_{\omega_{j}}\left(b_{j}\right)=r_{j}$ invertible in $R$ for some $j$. Then $\omega_{j}$ and $r_{j}^{-1} b_{j}$ forms a pair as above and we conclude that $\Omega \cong R$. Thus,

Proposition 3.9. If $R$ is a local ring, then $\Omega(A) \cong R$ for any central separable $R$-algebra $A$.

The standard model of a central separable algebra is as follows: Let $X$ and $Y$ be $R$-modules and let $\lambda: Y \otimes_{R} X \rightarrow R$ be an $R$-module homomorphism which is surjective. Then $A=X \otimes_{R} Y$ becomes an $R$-algebra if we set $\left(x_{1} \otimes y_{1}\right) \cdot\left(x_{2} \otimes y_{2}\right)=\lambda\left(y_{1} \otimes x_{2}\right)\left(x_{1} \otimes y_{2}\right)$.

If we define $\omega: A \otimes_{R} A \rightarrow A \otimes_{R} A$ by $\omega\left(\left(x_{1} \otimes y_{1}\right) \otimes\left(x_{2} \otimes y_{2}\right)\right)=$ $\left(x_{1} \otimes y_{2}\right) \otimes\left(x_{2} \otimes y_{1}\right)$, then $\omega \in \Omega(A)$ and $\operatorname{tr}_{\omega}(x \otimes y)=\lambda(y \otimes x)$. The surjectivity assumption shows that there exists $b \in A$ with $\operatorname{tr}_{\omega}(b)=1$. Thus, Proposition 3.8 implies that $A$ is central separable.

By the paragraph preceding Proposition 3.9, $A$ has the property that $\Omega(A) \cong R$ since $\operatorname{tr}_{\omega}(b)=1$.

Definition 3.2. The algebra described above will be called the $R$-elementary algebra determined by the triple $(X, Y, \lambda)$.

Proposition 3.10. Let $A$ be an R-algebra. Then $A$ is central separable algebra if and only if there is an $R$-algebra $B$, having $R$ as an $R$-module direct summand, such that $A \otimes_{R} B$ is isomorphic to an $R$-elementary algebra.

Proof. Suppose $A$ is central separable and consider the separator $\theta: A^{p} \otimes \Omega_{R} \rightarrow A^{e}$. We have

$$
\begin{aligned}
\theta(a \otimes b \otimes \omega) \cdot \theta\left(c \otimes d \otimes \omega^{\prime}\right) & =\theta\left(\theta(a \otimes b \otimes \omega) \cdot c \otimes d \otimes \omega^{\prime}\right) \\
& =\theta\left(\pi \circ \theta(a \otimes b c \otimes \omega) \otimes d \otimes \omega^{\prime}\right) \\
& =\theta\left(\operatorname{tr}(b c \otimes \omega) a \otimes d \otimes \omega^{\prime}\right) .
\end{aligned}
$$


Hence, $\theta$ is an algebra isomorphism with image $A^{e}$ and with domain the $R$-elementary algebra determined by $A, A \otimes_{R} \Omega$, and the pairing $\langle c, b \otimes \omega\rangle=\operatorname{tr}(b c \otimes \omega)=\operatorname{tr}_{\omega}(b c)$. Thus, $B=A^{o p}$ is an algebra for which $A \otimes_{R} B$ is isomorphic to an elementary algebra. Note that $A^{o p}$ contains $R$ as an $R$-module direct summand by Proposition 2.5.

Conversely, suppose $B$ is an $R$-algebra having $R$ as an $R$-module direct summand and suppose $A \otimes_{R} B$ is isomorphic to an elementary algebra. Then $A \otimes_{R} B$ is central separable. We shall show that this implies that $A$ is central separable.

We have $R$-module homomorphisms $\mu: B \rightarrow R$ and $\nu: R \rightarrow B$ with $\mu \circ \nu=1$. Then $1 \otimes \mu: A \otimes_{R} B \rightarrow A$ and $1 \otimes \nu: A \rightarrow A \otimes_{R} B$ exhibit $A$ as an $A$-bimodule direct summand of $A \otimes_{R} B$. Using this it is easy to see that $\left(A \otimes_{R} B\right)^{2}=A \otimes_{R} B$ implies $A^{2}=A$ and that $M\left(A \otimes_{R} B\right) \neq\left(A \otimes_{R} B\right)$ for each proper ideal $M$ of $R$ implies the same is true of $A$. To prove $A$ is $A^{e}$-projective, let $\alpha: M \rightarrow N$ and $\beta: A \rightarrow N$ be $A^{e}$-module homomorphisms with $\alpha$ surjective. On tensoring with $B$ we get $\left(A \otimes_{R} B\right)^{e}$-module homomorphisms $\alpha \otimes$ 1: $A \otimes_{R} B \rightarrow N \otimes_{R} B$ and $\beta \otimes 1: A \otimes_{R} B \rightarrow N \otimes_{R} B$. Since $\left(A \otimes_{R} B\right)$ is $\left(A \otimes_{R} B\right)^{e}$-projective we have a map $\gamma: A \otimes_{R} B \rightarrow M \otimes_{R} B$ with $(\alpha \otimes 1) \circ \gamma=(\beta \otimes 1)$. Then $\delta=(1 \otimes \mu) \circ \gamma \circ(1 \otimes \nu): A \rightarrow M$ is $A^{e}$-module homomorphism such that $\alpha \circ \gamma=\beta$. Thus, $A$ is projective.

To prove that $A$ is central, note that if $z \in Z(A)$ then $z \otimes 1 \epsilon$ $Z\left(A \otimes_{R} B\right)$ and, hence, is multiplication by an element of $R$. Using the fact that $A$ is an $R$-module direct summand of $A \otimes_{R} B$ shows that $z$ is multiplication by the same element of $R$.

4. The extended Brauer group. The classical Brauer group for $R$ is constructed from the class of central separable $R$-algebras with identity by declaring two such algebras, $A$ and $B$, to be equivalent if there is an algebra isomorphism $A \otimes_{R} \operatorname{Hom}_{R}(N, N) \rightarrow$ $B \otimes_{R} \operatorname{Hom}_{R}(M, M)$, where $N$ and $M$ are finitely generated projective faithful $R$-modules. The equivalence classes then form a group under tensor product. For central separable algebras the above equivalence relation agrees with Morita equivalence (cf. [4], Ch. 2). We shall use Morita equivalence as the starting point for our development of the extended Brauer group.

Let $A$ and $B$ be regular $R$-algebras $\left(A \otimes_{A} A=A\right.$ and $\left.B \otimes_{B} B \cong B\right)$. We declare $A$ and $B$ to be Morita equivalent and write $A \sim B$ provided there exists a regular left $A$-right $B$-module $M$ and a regular left $B$-right $A$-module $N$ such that $A \cong M \otimes_{B} N$ as an $A$-bimodule and $B \cong N \otimes_{A} M$ as a $B$-bimodule. It is a simple matter to prove that this is an equivalence relation, but the proof uses the regularity of all the modules involved. For example, reflexivity is just the regularity condition on $A: A \cong A \otimes_{A} A$. 
Given a Morita equivalence, the implementing modules $N$ and $M$ determine functors $X \rightarrow N \otimes_{A} X$ and $Y \rightarrow M \otimes_{B} Y$ which yield an equivalence between the category of regular left $A$-modules and the category of regular left $B$-modules. Half the proof that this is an equivalence is the sequence of isomorphisms

$$
M \otimes_{B}\left(N \otimes_{A} X\right) \cong\left(M \otimes_{B} N\right) \otimes_{A} X \cong A \otimes_{A} X \cong X
$$

(note the use of the regularity of $X$ ). The other half goes the same way. By interchanging $M$ and $N$ and tensoring on the right we get an equivalence between regular right $A$-modules and regular right $B$-modules. Tensoring on both sides yields an equivalence between $A$ and $B$-bimodules.

Given a Morita equivalence $A \sim B$ as above, the isomorphism $N \otimes_{A} M \rightarrow B$ determines a $B$-bimodule map $\lambda: N \otimes_{R} M \rightarrow B$ satisfying $\lambda(n a \otimes m)=\lambda(n \otimes a m)$. The pair $N, M$ and the map $\lambda$ determine the algebra $A$ as follows: $A$ is $M \otimes_{B} N$ as an $R$-module and the multiplication is determined by $\lambda$ as

$$
\begin{aligned}
\left(m_{1} \otimes_{B} n_{1}\right)\left(m_{2} \otimes_{B} n_{2}\right) & =\left(m_{1} \otimes_{B} n_{2}\right) \otimes_{A}\left(m_{2} \otimes_{B} n_{2}\right) \\
& =m_{1} \otimes_{B}\left(n_{1} \otimes_{A} m_{2}\right) \otimes_{B} n_{2}=m_{1} \otimes_{B} \lambda\left(n_{1} \otimes m_{2}\right) n_{2} .
\end{aligned}
$$

This is just like the construction of an $R$-elementary algebra, except that the ground ring is replaced by $B$.

On the other hand, suppose we are given a regular $R$-algebra $B$, a left $B$-module $N$, a right $B$-module $M$, and a $B$-bimodule map $\lambda: N \otimes_{R} M \rightarrow B$. We can define an algebra $A$ which is $M \otimes_{B} N$ as an $R$-module and with product given by

$$
\left(m_{1} \otimes_{B} n_{1}\right)\left(m_{2} \otimes_{B} n_{2}\right)=m_{1} \otimes_{B} \lambda\left(n_{1} \otimes m_{2}\right) n_{2} .
$$

Is $A$ then Morita equivalent to $B$ with $N$ and $M$ the implementing modules? The answer is yes if $B$ is central separable. The only thing that needs proving is that $\lambda: N \otimes_{R} M \rightarrow B$ induces a $B$-bimodule isomorphism $N \otimes_{A} M \rightarrow B$. We shall prove this using the separator $\theta$ defined in the previous section. First, we need a proposition which characterizes the trace map.

Proposition 4.1. Let $A$ be a central separable $R$-algebra and let $\Omega=\Omega(A)$, then:

(a) $0 \rightarrow[A, A] \otimes_{R} \Omega \rightarrow A \otimes_{R} \Omega \stackrel{\operatorname{tr}}{\rightarrow} R \rightarrow 0$ is a split short exact sequence of $R$-modules.

(b) If $T: A \otimes_{R} \Omega \rightarrow R$ is any surjective $R$-module homomorphism with kernel containing $[A, A] \otimes_{R} \Omega$, then $T=r \operatorname{tr}$ for an invertible element $r \in R$. 
Proof. Since $\operatorname{tr}(a b \otimes \omega)=\operatorname{tr}(b a \otimes \omega)$, ker tr clearly contains $[A, A] \otimes_{R} \Omega$.

To prove the reverse containment, we consider the maps $S(a \otimes b)=$ $b \otimes a$ on $A \otimes_{R} A$, and $S_{\theta}=\theta^{-1} \circ S \circ \theta$ and $S_{0}=S \otimes 1$ on $A \otimes_{R} A \otimes_{R} \Omega$. Since $S_{\theta} \circ S_{0}^{-1}$ is an $A^{e}$-bimodule automorphism of $A^{e} \otimes_{R} \Omega$, it must be given by an invertible element $r \in R$; i.e., $S_{\theta}=r S_{0}$.

With $\pi: A \otimes_{R} A \rightarrow A$ the multiplication map, recall that $1 \otimes \operatorname{tr}=$ $\pi \circ \theta$. Thus $\pi=(1 \otimes \operatorname{tr}) \circ \theta^{-1}$ and for $a, b \in A$ we have:

$$
[a, b]=(\pi-\pi \circ S)(a \otimes b)=(1 \otimes \operatorname{tr})\left(1-r S_{0}\right) \theta^{-1}(a \otimes b) .
$$

It follows that $(1 \otimes \operatorname{tr})\left(1-r S_{0}\right): A \otimes_{R}\left(A \otimes_{R} \Omega\right) \rightarrow A$ has $[A, A]$ as image. On tensoring with $\Omega$, we obtain a map $(1 \otimes \operatorname{tr})\left(1-r S_{1}\right)$ : $\left(A \otimes_{R} \Omega\right) \otimes_{R}\left(A \otimes_{R} \Omega\right) \rightarrow A \otimes_{R} \Omega$ with $[A, A] \otimes_{R} \Omega$ as image. Here, $S_{1}\left(a \otimes \omega_{1} \otimes b \otimes \omega_{2}\right)=b \otimes \omega_{1} \otimes a \otimes \omega_{2}$. If we choose $\beta \in A \otimes \otimes_{R} \Omega$ with $\operatorname{tr}(\beta)=1$, then $\gamma(\alpha)=(1 \otimes \operatorname{tr})\left(1-r S_{1}\right)(\alpha \otimes \beta)$ defines an $R$-module homomorphism $\gamma: A \otimes_{R} \Omega \rightarrow A \otimes_{R} \Omega$ which is the identity on ker tr and has $[A, A] \otimes_{R} \Omega$ as image. Since $[A, A] \otimes_{R} \Omega \subset$ ker tr, we conclude the two are equal. The map $\gamma$ also shows the sequence is $R$-split and (a) is proved.

To prove (b) note that if $T: A \otimes_{R} \Omega \rightarrow R$ has $\operatorname{ker} \operatorname{tr} \subset \operatorname{ker} T$ and is surjective, then it induces an $R$-module surjection $\left(A \otimes_{R} \Omega\right) / \operatorname{ker} \operatorname{tr} \rightarrow$ $R$. Since $\left(A \otimes_{R} \Omega\right) /$ ker tr $\cong R$ this map has no choice but to be multiplication by an invertible element $r \in R$. Then $T=r \mathrm{tr}$.

Returning to the discussion preceding Proposition 4.1, if $A=$ $M \otimes_{B} N$ is the algebra determined by regular left (resp. right) $B$ modules $N$ (resp. $M$ ) and a surjective $B$-bimodule homomorphism $\lambda: N \otimes_{R} M \rightarrow B$, then we shall call $A$ a $B$-elementary algebra.

Proposition 4.2. Suppose $B$ is central separable. Then an $R$ algebra $A$ is B-elementary if and only if it is Morita equivalent to $B$. In this case $A$ is also central separable.

Proof. We have already established that a Morita equivalence beteen $A$ and $B$ presents $A$ as a $B$-elementary algebra. The converse will follow if we can show that if $N, M$, and $\lambda$ present $A$ as a $B$ elementary algebra then the kernel of $\lambda: N \otimes_{R} M \rightarrow B$ is the kernel of $N \otimes_{R} M \rightarrow N \otimes_{A} M$. This will prove $N \otimes_{A} M \cong B$ and also establish the regularity of $N$ and $M$ as $A$-modules, since $A \otimes_{A} M=$ $M \otimes_{B}\left(N \otimes_{A} M\right)$ and $N \otimes_{A} A=\left(N \otimes_{A} M\right) \otimes_{B} N$.

First, $M$ and $N$ are $A$-modules in the obvious way: if $a=$ $m \otimes n$ then $a m^{\prime}=m \lambda\left(n \otimes m^{\prime}\right)$ and $n^{\prime} a=\lambda\left(n^{\prime} \otimes m\right) n$ for $m^{\prime} \in M$ and $n^{\prime} \in N . \quad$ Note that $n^{\prime} a \otimes m^{\prime}-n^{\prime} \otimes a m^{\prime}=\lambda\left(n^{\prime} \otimes m\right) n \otimes m^{\prime}-n^{\prime} \otimes$ $m \lambda\left(n \otimes m^{\prime}\right)$ which is in the kernel of $\lambda: N \otimes_{R} M \rightarrow B$. Hence, the kernel of $\lambda$ contains the kernel of $N \otimes_{R} M \rightarrow N \otimes_{A} M$. 
Now consider the separator $\theta: B \otimes_{R} B \otimes_{R} \Omega \rightarrow B \otimes_{R} B$ for $B$. It is an isomorphism for the two left and two right $B$-module actions but interchanges the two right actions. Thus, if we apply $M \otimes_{B}(\quad)$ to the second factor in both domain and range and $\left(\quad \otimes_{B} N\right.$ to the second factor in the domain and the first factor in the range, we obtain a map

$$
\tilde{\theta}: B \otimes_{R} A \otimes_{R} \Omega \longrightarrow N \otimes_{R} M
$$

which is an isomorphism for the two $A$ actions and the two $B$ actions but interchanges the right actions. Hence, for each $\alpha \epsilon$ $A \otimes_{R} \Omega, b \rightarrow \lambda \circ \tilde{\theta}(b \otimes \alpha): B \rightarrow B$ is a member of $Z(B)$. Since $B$ is central $\lambda \circ \tilde{\theta}(b \otimes \alpha)=T(\alpha) b$ for an $R$-module map $T: A \otimes_{R} \Omega \rightarrow R$. Note $T$ is surjective because $\tilde{\theta}$ and $\lambda$ are surjective. Then kernel of $T$ contains $[A, A] \otimes_{R} \Omega$ because of the $A$-module properties of $\tilde{\theta}$ and the fact that $\lambda(n a \otimes m-n \otimes a m)=0$ for $a \in A, n \in N, m \in M$.

Assume for the moment that $A$ is central separable and the module $\Omega=\Omega(B)$ is isomorphic to $\Omega(A)$. Then Proposition 4.1 implies that $T$ is an $R$ multiple of the trace for $A$. Since $\lambda=(1 \otimes T) \circ \tilde{\theta}^{-1}$, its kernel is $\theta \operatorname{ker}(1 \otimes T)$. By Proposition 4.1 the kernel of $T$ is $[A, A] \otimes_{R} \Omega$ and $\tilde{\theta}$ maps $B \otimes_{R}[A, A] \otimes_{R} \Omega$ exactly to the kernel of $N \otimes_{R} M \rightarrow N \otimes_{a} M$. Thus, the proof is finished if we can show that $A$ is central separable and $\Omega(B) \cong \Omega(A)$.

Consider the sequence of maps

$$
B \otimes_{R} A \otimes_{R} \Omega \stackrel{\tilde{\theta}}{\longrightarrow} N \otimes_{R} M \stackrel{\lambda}{\longrightarrow} B
$$

$\tilde{\theta}$ is a $B$-bimodule isomorphism and an $A$ bimodule isomorphism while $\lambda$ is a $B$-bimodule homomorphism. If we apply $M \otimes_{B}(\quad) \otimes_{B} N$ we obtain the pair of maps

$$
A \otimes_{R} A \otimes_{R} \Omega \stackrel{\theta^{A}}{\longrightarrow} A \otimes_{R} A \stackrel{\pi^{A}}{\longrightarrow} A
$$

where $\pi^{A}$ is the multiplication in $A$. Also, since $\lambda \circ \widetilde{\theta}=1 \otimes T: B \otimes \bigotimes_{R}$ $\left(A \otimes_{R} \Omega\right) \rightarrow B$ we conclude that $\pi^{A} \circ \theta^{A}=1 \otimes T: A \otimes_{R}\left(A \otimes_{R} \Omega\right) \rightarrow A$.

Now $\theta^{A}$ has the properties of the separator for $A$ except that we don't know a priori that $\Omega(A) \cong \Omega(B)$. However, if $\omega \in \Omega=\Omega(B)$ then $\tilde{\omega}\left(a \otimes a^{\prime}\right)=\theta^{A}\left(a \otimes a^{\prime} \otimes \omega\right)$ clearly defines an element $\tilde{\omega} \in \Omega(A)$. Thus, $\omega \rightarrow \tilde{\omega}$ maps $\Omega(B)$ into $\Omega(A)$ and $\theta^{A}$ is the separator for $A$ composed with the map $A \otimes_{R} A \otimes_{R} \Omega \rightarrow A \otimes_{R} A \otimes_{R} \Omega(A)$ induced by $\omega \rightarrow \tilde{\omega}$. From this it follows that $T$ is the composition of the trace for $A$ with the induced map $A \otimes_{R} \Omega \rightarrow A \otimes_{R} \Omega(A)$. Since $T$ has image $R$, it follows from Proposition 3.8 that $A$ is central separable and $\omega \rightarrow \tilde{\omega}$ is surjective. Since $\Omega$ is an invertible $R$-module $\omega \rightarrow \tilde{\omega}$ must be an isomorphism. 
The previous proposition is a powerful tool for generating Morita equivalences. For example, it easily yields:

Proposition 4.3. Let $N$ be a regular left ideal and $M$ a regular right ideal of the central separable algebra $A$. If $N M=A$ then the subalgebra $M N$ is central separable and Morita equivalent to $A$.

Proof. Let $\lambda: N \otimes_{R} M \rightarrow N M=A$ be multiplication in $A$. From Proposition 4.2 (with $B$ replaced by $A$ ) we have that the $A$-elementary algebra $M \otimes_{A} N$, with product determined by $\lambda$, is central separable and Morita equivalent to $A$. If we can prove that $\pi: M \otimes_{A} N \rightarrow M N$ is bijective, then the proof will be complete since the multiplication induced by $\lambda$ will then agree with the product in $M N$ as a subalgebra of $A$.

Clearly $\pi$ is surjective. To prove it is injective consider the map $\varphi_{N}: N \rightarrow A \otimes{ }_{R} N$ of Proposition 1.2.

It is a left $A$-module homomorphism and a right inverse for the multiplication map $A \otimes_{R} N \rightarrow N$. Thus, if $\sum m_{i} n_{i}=0$ and $\varphi_{N}\left(n_{i}\right)=\sum_{j} a_{i j} \otimes n_{i j}$ we have $\sum_{i} m_{i} \sum_{j} a_{i j} \otimes n_{i j}=\varphi_{N}\left(\sum m_{i} n_{i}\right)=0$ and so

$$
\sum m_{i} \otimes n_{i}=\sum_{i j}\left(m_{i} \otimes a_{i j} n_{i j}-m_{i} a_{i j} \otimes n_{i j}\right)
$$

which belongs to $\left.\operatorname{ker} M \otimes_{R} N \rightarrow M \otimes_{A} N\right)$. It follows that $M \otimes_{A} N \rightarrow$ $M N$ is injective.

Corollary 4.1. Every central separable R-algebra $A$ is Morita equivalent to a subalgebra which is contained in a finitely generated $R$-submodule of $A$.

Proof. If $a, b, c \in A$ then $a c b=\sum \operatorname{tr}\left(v_{i} c \otimes \omega_{i}\right) n_{i}$ where

$$
\sum n_{i} \otimes v_{i} \otimes \omega_{i}=\theta^{-1}(a \otimes b) \in A \otimes_{R} A \otimes_{R} \Omega \text {. }
$$

It follows that if $M$ and $N$ are finitely generated ideals then $M N$ is contained in a finitely generated $R$-submodule of $A$. Now suppose $a_{i}, b_{i}, c_{i}$ and $\omega_{i}$ are chosen $(i=1, \cdots, n)$ so that $\operatorname{tr}\left(\sum a_{i} b_{i} c_{i} \otimes \omega_{i}\right)=1$. Then $\pi \circ \theta\left(A \otimes \sum a_{i} b_{i} c_{i} \otimes \omega_{i}\right)=A$ but is contained in $\left(\sum A c_{i}\right) \cdot\left(\sum a_{i} A\right)$. Thus $N=\sum A c_{i}$ and $M=\sum a_{i} A$ are finitely generated regular left (resp. right) ideals satisfying $N M=A$. By the proposition, $A \sim$ $M N$ and $M N$ is contained in a finitely generated $R$-submodule of $A$.

COROLLARY 4.2. If $R$ is noetherian then every central separable $R$-algebra is Morita equivalent to a subalgebra which is a finite $R$ module. 
It is apparent from Corollary 4.1 that the Morita equivalence classes of central separable $R$-algebras form a set. It is also clear from the definition that algebra tensor product induces a well defined operation on this set under which it is an abelian semigroup. The ground ring $R$ determines an identitiy for this semigroup. By Proposition 3.10 , if $A$ is a central separable $A$-algebra then there is another $R$-algebra $B$, necessarily central separable by the same proposition, such that $A \otimes_{R} B$ is isomorphic to an $R$-elementary algebra. By Proposition 4.2 an $R$-elementary algebra is Morita equivalent to $R$. Thus, our semigroup is actually an abelian group.

Definition 4.1. The extended Brauer group of $R$, denoted $\widetilde{B}(R)$, is defined to be the group of Morita equivalence classes of central separable $R$-algebras. If $A$ is a central separable $R$-algebra, we denote its class in $\widetilde{B}(R)$ by $[A]$.

Equality in the extended Brauer group can also be defined in a way that is more in the spirit of Auslander-Goldman [2]:

Proposition 4.4. If $A$ and $B$ are central separable $R$-algebras, then $[A]=[B]$ in $\widetilde{B}(R)$ if and only if there are $R$-elementary algebras $E$ and $F$ so that $A \otimes_{R} E \cong B \otimes_{R} F$.

Proof. Since $[E]=[R]=[F]$ we have $[A]=\left[A \otimes_{R} E\right]=$ $\left[B \otimes_{R} F\right]=[B]$.

Conversely, if $[A]=[B]$ then $\left[A \otimes_{R} B^{0}\right]=[A][B]^{-1}=[R]$ and $\left[B^{0} \otimes_{R} B\right]=[B]^{-1}[B]=[R]$ so that $E=B^{0} \otimes_{R} B$ and $F=A \otimes_{R} B^{0}$ are $R$-elementary algebras. However,

$$
A \otimes_{R} E=A \otimes_{R} B^{0} \otimes_{R} B \cong B \otimes_{R} A \otimes_{R} B^{0}=B \otimes_{R} F .
$$

The usual Brauer group $B(R)$ is defined in a similar way except that only algebras with identity are considered and equality is defined, as in Proposition 4.4, but with $R$-elementary algebras replaced by algebras of the form $\operatorname{Hom}_{R}(M, M)$ where $M$ is a faithful finitely generated projective $R$-module. Now such an algebra is $R$-elementary in our sense. In fact, $\operatorname{Hom}_{R}(M, M) \cong M \otimes_{R} M^{\prime}$ where $M^{\prime}=\operatorname{Hom}_{R}(M, R)$ and multiplication is defined by the usual pairing $\lambda\left(m^{\prime} \otimes m\right)=m^{\prime}(m)$. On the other hand we have:

Proposition 4.5. If $E=M \otimes_{R} N$ is an R-elementary algebra with an identity, then $M$ is faithful, finitely generated and projective, $N \cong M^{\prime}$ and $E \cong \operatorname{Hom}_{R}(M, M)$.

Proof. Let $\lambda: N \otimes_{R} M \rightarrow R$ be the map inducing multiplication in $E$. The fact that $\lambda$ is surjective implies that $N$ and $M$ are 
faithful $R$-modules. If $e=\sum m_{i} \otimes n_{i}$ is an identity for $E$, then

$$
m \otimes n=e(m \otimes n)=\sum \lambda\left(n_{i} \otimes m\right) m_{i} \otimes n .
$$

Thus, if $k \in N \otimes_{R} M$ is chosen so that $\lambda(k)=1$, we have $m \otimes k=$ $\sum \lambda\left(n_{i} \otimes m\right) m_{i} \otimes k$ and, on applying $1 \otimes \lambda, m=\sum \lambda\left(n_{i} \otimes m\right) m_{i}$. Then the maps $m \rightarrow\left\{\lambda\left(n_{i} \otimes m\right)\right\}: M \rightarrow R^{n}$ and $\left\{r_{i}\right\} \rightarrow \sum r_{i} m_{i}$ express $M$ as an $R$-module direct summand of $R^{n}$. Hence, $M$ is finitely generated and projective and every element of $M^{\prime}$ has the form $m \rightarrow$ $\sum r_{i} \lambda\left(n_{i} \otimes m\right)=\lambda(n \otimes m)$ with $n=\sum r_{i} n_{i}$. The analogous argument, using the fact that $e$ is a right identity, shows that $n \rightarrow \sum \lambda\left(n \otimes m_{i}\right)$ : $N \rightarrow R^{n}$ embeds $N$ as a direct summand of $R^{n}$ and completes the proof that the form $(n, m) \rightarrow \lambda(n \otimes m)$ defines an isomorphism of $N$ with $M^{\prime}$. Thus, $E \cong M \otimes_{R} M^{\prime} \cong \operatorname{Hom}_{R}(M, M)$.

If $A$ and $B$ are central separable $R$-algebras with identity which determine the same class in $\widetilde{B}(R)$, then $A \otimes_{R} B^{0}$ is an $R$-elementary algebra with identity and, hence, has the form $\operatorname{Hom}_{R}(M, M)$ for a faithful, finitely generated projective $R$-module $M$. This implies that $A$ and $B$ determine the same element of the classical Brauer group $B(R)$. Thus, we have:

Proposition 4.6. The classical Brauer group $B(R)$ is embedded as a subgroup of $\widetilde{B}(R)$.

Just as in the classical case, $\widetilde{B}$ is a covariant functor on the category of rings and identity preserving ring homomorphisms. For such a homomorphism $R \rightarrow S, A \rightarrow A \otimes{ }_{R} S$ determines the corresponding group homomorphism $\widetilde{B}(R) \rightarrow \widetilde{B}(S)$.

We now proceed with an analogue of the classical result that $B(R)=(0)$ if $R$ is a Henselian local ring with maximal ideal $M$ and $R / M$ is algebraically closed (cf. [2], Prop. 6.1). The proof rests on the existence of rank one idempotents, where an idempotent $p \in A$ is called rank one if $p A p=R p \cong R$. An idempotent $p$ is called nondegenerate if $p \notin M A$ for each maximal ideal $M$ of $R$. Note that a rank one idempotent $p$ is nondegenerate. Otherwise, we would have $p \in M A$ and $R p=p A p \subset M p A p=M p$. Since $R \rightarrow R p$ is an $R$ module isomorphism, this is impossible.

Proposition 4.3. If $A$ is a central separable $R$-algebra containing a nondegenerate idempotent $p$, then $[A]=[p A p]$ and $[A] \in B(R)$. If $p$ is rank one then $[A]=(0)$.

Proof. If we can show that $A p A=A$ if $p$ is a nondegenerate idempotent, it will follow from Proposition 4.3, applied to the ideals 
$A p$ and $p A$, that $A$ is Morita equivalent to $p A p$. Since $p A p$ has identity $p,[p A p]$ belongs to $B(R)$. If $p$ has rank one $p A p \cong R$ and $[A]=(0)$.

If $A p A \neq A$ it is contained in $M A$ for some maximal ideal $M \subset$ $R$ by Proposition 3.5. This implies $p \in M A$ in violation of the nondegeneracy hypothesis.

Proposition 4.8. If $R$ is an algebraically closed field, then each central separable $R$-algebra contains a rank one idempotent and, hence, $\widetilde{B}(R)=(0)$.

Proof. By Corollary 4.2 may assume $A$ is finite dimensional. If we can show $A$ contains a nonzero idempotent $p$, then $p A p$ will be a central separable algebra with identity and, hence, a matrix algebra over $R$ by the classical theory. Then $p A p$ and, therefore $A$, contains a rank one idempotent.

Now $A$ contains a nonnilpotent element $a$. Otherwise $A$ would be a nilpotent algebra, since it is finite dimensional, violating $A^{2}=$ $A$. Then by standard matrix theory, some polynomial in $L_{a}$ is a nonzero idempotent, where $L_{a}: A \rightarrow A$ is left multiplication by $a$. That is, there exists $b \in A$ with $L_{b}^{2}=L_{b} \neq(0)$. If $p=b^{2}$ then $p$ is our nonzero idempotent.

Proposition 4.9. If $R$ is a Henselian ring with maximal ideal $M$ and $R / M$ is algebraically closed, then each central separable $R$ algebra contains a rank one idempotant and, hence, $\widetilde{B}(R)=(0)$.

Proof. A Henselian ring is a local ring $R$ such that whenever $B$ is a finite $R$-algebra, $I \subset B$ a two-sided ideal, and $p \in B / I$ an idempotent, then $p$ is the image of some idempotent in $B$. This is not the usual definition of Henselian ring, but is equivalent to it (cf. [3], p. 136).

By Proposition 2.7, $A / M A$ is central separable over the algebraically closed field $R / M$. Thus, $A$ contains an element $a$ such that the image of $a$ in $A / M A$ is a rank one idempotent. We shall show that $a$ is contained in a subalgebra of $A$ that is finitely generated as an $R$-module.

By Proposition 3.9 the separator $\theta$ may be regarded as a map from $A \otimes_{R} A$ to $A \otimes_{R} A$. We define $k_{a}: A \rightarrow A$ by $k_{a}(b)=a b a$ and note that $k_{a}(b)=\sum u_{i} \operatorname{tr}\left(v_{i} b\right)$ where $\theta\left(\sum u_{i} \otimes v_{i}\right)=a \otimes a$. In other words, $k_{a}=u \circ v$ where $u: R^{n} \rightarrow A$ and $v: A \rightarrow R^{n}$ are defined by $u\left(\left\{r_{i}\right\}\right)=\sum r_{i} u_{i}$ and $v(b)=\left\{\operatorname{tr}\left(v_{i} b\right)\right\}$. It follows that $k_{a}^{m}=u \circ w^{m-1} \circ v$ where $w=v \circ u: R^{n} \rightarrow R^{n}$. Since $w$ is integral over $R$ so is $k_{a}$ and we have $q\left(k_{a}\right)=0$ for some monic polynomial $q \in R[x]$. Then $q\left(k_{a}\right)(a)=$ 
0 is a monic polynomial equation satisfied by $a$. It follows that the subalgebra of $A$ generated by $a$ is a finitely generated $R$-module.

Since $R$ is Henselian, the subalgebra of $A$ generated by $a$ contains an idempotent $p$ equivalent to $a$ modulo $M A$. Now $p A p$ is a central separable algebra with identity and, hence, is a finitely generated projective $R$-module. Since $R$ is local, $p A p$ is a free $R$ module and, hence, one dimensional since it is one dimensional modulo $M$. Thus, $p$ has rank one.

5. Automorphisms. In the classical theory of central separable algebras with identity one has the following exact sequence (cf. [8], Ch. II, §6)

$$
0 \longrightarrow \operatorname{Inn}(A) \longrightarrow \operatorname{Aut}_{R}(A) \longrightarrow \operatorname{Pic}(R) \longrightarrow Q_{B}(M) \longrightarrow 0
$$

where $\operatorname{Aut}_{R}(A)$ is the group of $R$-algebra automorphisms of $A, \operatorname{Inn}(A)$ is the group of inner automorphisms of $A, B$ is a central separable $R$-algebra Morita equivalent to $A, M$ is a projective right $B$-module and $A$ is the central separable $R$-algebra $\operatorname{Hom}_{B}(M, M)$. The group $Q_{B}(M)$ is constructed from isomorphism classes of projective right $B$-modules $N$ for which $\operatorname{Hom}_{B}(N, N) \cong A$. In effect, this classifies both the automorphisms of $A$ and the distinct ways in which $A$ may be presented as a $B$-elementary algebra. In this section, we obtain essentially the same results, with appropriate modification, in the setting of algebras without identity.

Let $A$ be a central separable $R$-algebra and let $\operatorname{Aut}_{R}(A)$ denote its group of $R$-algebra automorphisms. If $\sigma \in \operatorname{Aut}_{R}(A)$ then $A_{\sigma}$ will denote the left $A^{e}$-module consisting of $A$ with $A^{e}$-module action defined by $(a \otimes b) \cdot c=a c \sigma(b)$. Then we set $P_{\sigma}=\operatorname{Hom}_{A^{e}}\left(A, A_{\sigma}\right)$. Note that if $\sigma=1$ then $P_{o}=Z(A)=R$.

The $R$-module $P_{o}$ is always nontrivial. In fact, if $\omega \in \Omega(A)$ then for each $b \in A$

$$
a \longrightarrow \pi \circ(1 \otimes \sigma) \circ \omega(a \otimes b)
$$

defines an element of $P_{\sigma}$. By Proposition 3.7, if $\omega$ ranges over $\Omega(A), b$ over $A$, then the images of the resulting elements of $P_{\sigma}$ will span $A_{\sigma}$. Thus,

Proposition 5.1. For each $\sigma \in \operatorname{Aut}_{R}(A), P_{\sigma} \cdot A=A_{\sigma}$.

Now let $\sigma, \tau$ be elements of $\operatorname{Aut}_{R}(A)$. If $f \in P_{\sigma}$ and $g \in P_{\tau}$ are regarded as maps from $A$ to $A$, they may be composed. Then

$$
f \circ g(a c b)=f(a g(c) \tau(b))=a f \circ g(c) \sigma \circ \tau(b) .
$$


Hence, $f \circ g \in P_{\sigma \circ \tau}$ and we have an $R$-module homomorphism $P_{\sigma} \otimes_{R} P_{\tau} \rightarrow$ $P_{o \circ \tau}$.

Proposition 5.2. The map $P_{\sigma} \otimes_{R} P_{\tau} \rightarrow P_{\sigma \circ}$ is an isomorphism for each $\sigma$ and $\tau$ in $\operatorname{Aut}_{R}(A)$.

Proof. We first prove that $P_{\sigma} \otimes_{R} P_{\sigma^{-1}} \rightarrow P_{1}=R$ is an isomorphism. If it were not surjective its image would lie in a maximal ideal $M$ of $R$. However, $P_{\sigma} \cdot A=A, P_{\sigma^{-1}} \cdot A=A$, and $M A \neq A$ make this impossible. Thus, let $\left\{u_{\imath}\right\} \subset P_{\sigma}$ and $\left\{v_{i}\right\} \subset P_{\sigma^{-1}}$ be chosen so that $\sum u_{i} v_{i}=1$. Then $r \rightarrow \sum r u_{i} \otimes v_{i}: R \rightarrow P_{\sigma} \otimes_{R} P_{\sigma^{-1}}$ provides an inverse for $P_{\sigma} \otimes_{R} P_{\sigma^{-1}} \rightarrow R$. That it is a right inverse is clear. It is also a left inverse since, for $u \in P_{\sigma}$ and $v \in P_{\sigma^{-1}}$, we have $v u_{i} \in R$ and, thus,

$$
\sum(u v) u_{\imath} \otimes v_{\imath}=\sum u \otimes\left(v u_{i}\right) v_{i}=(u \otimes v) \sum u_{\imath} v_{\imath}=u \otimes v .
$$

Now for $\tau \in \operatorname{Aut}_{R}(A)$ we have

$$
P_{\sigma \tau \tau} \cong R \otimes_{R} P_{\sigma \tau \tau} \cong P_{\sigma} \otimes_{R} P_{\sigma^{-1}} \otimes_{R} P_{\sigma \tau}
$$

and a map $P_{\sigma} \otimes_{R} P_{\sigma^{-1}} \otimes_{R} P_{\sigma \circ \tau} \rightarrow P_{\sigma} \otimes_{R R} P_{\tau}$ induced by composing the second and third factors. The resulting map $P_{\sigma \circ \sigma} \rightarrow P_{\sigma} \otimes_{R} P_{\tau}$ is clearly an inverse for the composition map.

The above proposition shows that $\sigma \rightarrow P_{\sigma}$ determines a homomorphism of $\operatorname{Aut}_{R}(A)$ into $\operatorname{Pic}(R)$, where $\operatorname{Pic}(R)$ is the group of isomorphism classes of $R$-modules which are invertible under $\otimes_{R}$.

Suppose that $P_{o}$ represents the trivial element of $\operatorname{Pic}(R)$, i.e., $P_{\sigma} \cong R$. This is equivalent to the existence of an $R$-module generator $u \in P_{\sigma}$. Then $P_{\sigma^{-1}}$ is also singly generated, by let's say $v$. Thus $u v=r \in R$ is invertible as is $v u=s$; furthermore $u v u=r u=s u$ and so $r=s$. It follows that $r^{-1} v=w$ has the property that $u w=w u=$ 1. Now $w(a b c)=a w(b) \sigma^{-1}(c)$ so that if $u^{\prime}=\sigma \circ w$ then $u^{\prime}(a b c)=$ $\sigma(a) u^{\prime}(b) c$ and $u^{\prime} \circ u=\sigma$.

Since $u^{\prime}$ commutes with right multiplication in $A$ and $u$ commutes with left multiplication in $A$, it makes notational sense to write $u^{\prime}(a)=u^{\prime} a$ and $u(a)=a u$. We will not get in trouble with this since left and right module endomorphisms commute on an algebra satisfying $A^{2}=A$. Then the equation $u^{\prime} \circ u=\sigma$ becomes $\sigma(a)=u^{\prime} a u$ for $a \in A$. We also have the relation $(a u)\left(u^{\prime} b\right)=a b$ for $a, b \in A$ since $\sigma(a b)=\sigma(a) \sigma(b)=\left(u^{\prime} a u\right)\left(u^{\prime} b u\right)=\sigma\left((a u)\left(u^{\prime} b\right)\right)$. This suggests the following definition:

Definition 5.1. An automorphism $\sigma$ of $A$ will be called inner if it has the form $\sigma(a)=u^{\prime} a u$, where $u$ is an automorphism of $A$ 
as a left $A$-module, $u^{\prime}$ is an automorphism of $A$ as a right $A$-module and $\left(a u^{\prime}\right)(u b)=a b$ for all $a, b \in A$. We denote the set of inner automorphisms by $\operatorname{Inn}(A)$.

Clearly $\operatorname{Inn}(A)$ is a subgroup of $\operatorname{Aut}(A)$. We showed above that the kernel of the map $\operatorname{Aut}_{R}(A) \rightarrow \operatorname{Pic}(R)$ is contained in this subgroup. Conversely, suppose $\sigma \in \operatorname{Inn}(A)$ so that $\sigma(a)=u^{\prime} a u$ with $u^{\prime}$ and $u$ as above. Then $u(a b c)=a b c u=(a b u)\left(u^{\prime} c u\right)=a u(b) \sigma(c)$ and, hence, $u$ belongs to $P_{\sigma}$. The fact that $u$ is invertible as a map from $A$ to $A$ implies that it is a generator for $P_{\sigma}$. Thus, $P_{o} \cong R$. This proves that $\operatorname{Inn}(A)$ is exactly the kernel of $\operatorname{Aut}_{R}(A) \rightarrow \operatorname{Pic}(R)$. Thus,

Proposition 5.3. If $A$ is a central separable $R$-algebra then there is an exact sequence

$$
0 \longrightarrow \operatorname{Inn}(A) \longrightarrow \operatorname{Aut}_{A}(A) \longrightarrow \operatorname{Pic}(R) \text {. }
$$

We propose now to characterize the cokernel of the last map in this sequence.

Let $B$ be an algebra Morita equivalent to $A$ (e.g., we could choose $B=A$ or $B=R$ if $A$ is $R$-elementary). Let $N, M$, and $\lambda: N \otimes_{R} M \rightarrow$ $B$ be data presenting $A$ as a $B$-elementary algebra. If $P$ is an invertible $R$-module with inverse $P^{-1}$, then $N^{P} \otimes_{R} M^{P} \cong N \bigotimes_{R} M$ where $N^{P}=N \otimes_{R} P$ and $M^{P}=M \otimes_{R} P^{-1}$. This isomorphism, composed with $\lambda$, yields a map $\lambda: N^{P} \otimes_{R} M^{P} \rightarrow B$. Hence, we have data for another $B$-elementary algebra $A^{P}=M^{P} \otimes_{B} N^{P}$. Clearly, the isomorphism $P^{-1} \otimes_{R} P \rightarrow R$ determines an isomorphism $\varphi: A^{P} \rightarrow A$. Thus, each invertible $R$-module $P$ transforms $(N, M, \lambda)$ into another set of data presenting $A$ as a $B$-elementary algebra.

It seems appropriate to call two sets of data $(N, M, \lambda)$ and $\left(N^{\prime}, M^{\prime}, \lambda^{\prime}\right)$ equivalent if there are $B$-module isomorphisms $\nu: N^{\prime} \rightarrow N$ and $\mu: M^{\prime} \rightarrow M$ such that $\lambda^{\prime}=\lambda \circ(\nu \otimes \mu)$. Clearly, the $B$-elementary algebras so determined are isomorphic in this case (via $\mu \otimes \nu$ ).

Proposition 5.4. With ( $N, M, \lambda), P$, and $\left(N^{P}, M^{P}, \lambda^{P}\right)$ as above, $(N, M, \lambda)$ and $\left(N^{P}, M^{P}, \lambda^{P}\right)$ are equivalent sets of data if and only if $P \cong P_{\sigma}$ for some $\sigma \in \operatorname{Aut}_{R}(A)$.

Proof. If $v \in P_{\sigma}$ and $u \in P_{\sigma}^{-1}$ then $v$ is a left $A$-module endomorphism of $A$ and $\sigma \circ u$ is a right $A$-module endomorphism of $A$. From this and $A \otimes_{A} M \cong M$ and $N \otimes_{A} A \cong N$ we conclude that there are well defined $B$-module homomorphisms $\nu: N^{P} \rightarrow N$ and $\mu: M^{P} \rightarrow M$ characterized by $\nu(n a \otimes v)=n v(a)$ and $\mu(a m \otimes u)=\sigma \circ u(a) m$. Using the isomorphism $P_{\sigma} \otimes_{R} P_{\sigma}^{-1} \rightarrow R$ one easily obtains inverses for these maps. For example, the inverse for $\nu$ is the composition of $N \rightarrow$ 
$N \otimes_{R} P_{\sigma}^{-1} \otimes_{R} P_{\sigma} \rightarrow N \otimes_{R} P_{\sigma}$ where the second map is induced by a map $N \otimes_{R} P_{\sigma}^{-1} \rightarrow N$ constructed just as $\nu$ was but with $P_{\sigma}$ replaced by $P_{\sigma}^{-1}$. Thus, $\mu$ and $\nu$ are $B$-module isomorphisms.

We must prove that $\lambda^{P}=\lambda \circ(\nu \otimes \mu)$. First, note that $\lambda^{P}=\lambda \circ \psi$ where $\psi$ is the isomorphism $\left(N \otimes_{R} P_{o}\right) \otimes_{R}\left(M \otimes_{R} P_{\sigma}^{-1}\right) \rightarrow N \otimes_{R} M$ induced by $P_{\sigma} \otimes_{R} P_{\sigma}^{-1} \rightarrow R$. Now $\psi^{-1}(n \otimes m)=\sum\left(n \otimes v_{i}\right) \otimes\left(m \otimes u_{i}\right)$ if $v_{i} \in P_{\sigma}$ and $u_{i} \in P_{\sigma}^{-1}$ are chosen so that $\sum v_{i} u_{i}=1$. Thus,

$$
\begin{aligned}
\lambda \circ(\nu \otimes \mu) \circ \psi^{-1}\left(n a \otimes a^{\prime} m\right) & =\lambda\left(\sum_{i} n v_{i}(a) \otimes\left(\sigma \circ u_{i}\right)\left(a^{\prime}\right) m\right) \\
& =\sum_{i} n v_{i}(a) \otimes_{A}\left(\sigma \circ u_{i}\right)\left(a^{\prime}\right) m \\
& =n \otimes_{A}\left(\sum v_{i}(a) \sigma \circ u_{i}\left(a^{\prime}\right)\right) m \\
& =n \otimes_{A}\left(\sum v_{i} u_{i}\left(a \alpha^{\prime}\right)\right) m=n \otimes_{A} a a^{\prime} m \\
& =n a \otimes_{A} a^{\prime} m=\lambda\left(n a \otimes a^{\prime} m^{\prime}\right) .
\end{aligned}
$$

Thus, $\lambda \circ(\nu \otimes \mu) \circ \psi^{-1}=\lambda$ and $\lambda^{P}=\lambda \circ(\mu \otimes \nu)$.

Conversely, suppose $\nu: N^{P} \rightarrow N$ and $\mu: M^{P} \rightarrow N$ are $B$-module isomorphisms such that $\lambda^{P}=\lambda \circ(\nu \otimes \mu)$. Then $\mu \otimes \nu: A^{P}=M^{P} \otimes_{B} N^{P} \rightarrow$ $M \otimes_{B} N$ is an algebra isomorphism. We already have an algebra isomorphism $\varphi: A^{P} \rightarrow A$ induced by the isomorphism $P^{-1} \otimes_{R} P \rightarrow R$. Thus, $(\mu \otimes \nu) \circ \varphi^{-1}=\sigma$ is an automorphism of $A$. To complete the proof, we must show that $P \cong P_{\sigma}$. For $p \in P$ we define $v_{p}(m \otimes n)=$ $m \otimes \nu(n \otimes p)$. Then $v_{p}$ maps $A=M \otimes_{B} N$ to itself and is a left $A$-module endomorphism. Also, with $v_{i} \in P, u_{\imath} \in P^{-1}$ chosen so that $\sum v_{i} u_{i}=1$,

$$
\begin{aligned}
v_{p}(m \otimes n) \sigma\left(m^{\prime} \otimes n^{\prime}\right) & =v_{p}(m \otimes n)(\mu \otimes \nu) \circ \varphi^{-1}\left(m^{\prime} \otimes n^{\prime}\right) \\
& =v_{p}(m \otimes n) \sum \mu\left(m^{\prime} \otimes u_{i}\right) \otimes \nu\left(n^{\prime} \otimes v_{i}\right) \\
& =m \otimes \sum \lambda\left(\nu(n \otimes p) \otimes \mu\left(m^{\prime} \otimes u_{i}\right)\right) \nu\left(n^{\prime} \otimes v_{i}\right) \\
& =m \otimes \sum \lambda^{p}(n \otimes p) \otimes\left(m^{\prime} \otimes u_{i}\right) \nu\left(n^{\prime} \otimes v_{i}\right) \\
& =m \otimes \sum \lambda\left(n \otimes p u_{i} m^{\prime}\right) \nu\left(n^{\prime} \otimes v_{i}\right) \\
& =m \otimes \lambda\left(n \otimes m^{\prime}\right) \nu\left(n^{\prime} \otimes p \sum u_{i} v_{\imath}\right) \\
& =m \otimes \lambda\left(n \otimes m^{\prime}\right) \nu\left(n^{\prime} \otimes p\right) \\
& =v_{p}(m \otimes n) \cdot\left(m^{\prime} \otimes n^{\prime}\right) .
\end{aligned}
$$

Thus, $v_{p}\left(a a^{\prime}\right)=v_{p}(a) \sigma\left(a^{\prime}\right)$ and $v_{p} \in P_{\sigma}$. Since $P$ and $P_{\sigma}$ are both invertible $R$-modules, the only way $p \rightarrow v_{p}: P \rightarrow P_{\sigma}$ could fail to be an isomorphism is if it has image in $I P_{\sigma}$ for some proper ideal $I$ of $R$. However, this would imply $I N=N$ and, via $\lambda, I B=B$. Since this is impossible, we have $P \cong P_{\sigma}$ and the proof is complete.

We next show that given one set $(N, M, \lambda)$ of data presenting $A$ as a $B$-elementary algebra, every other such set has the form $\left(N^{P}, M^{P}, \lambda^{P}\right)$ for some invertible $R$-module $P$. 
Given data $\left(N^{\prime}, M^{\prime}, \lambda^{\prime}\right)$ with $M^{\prime} \otimes_{B} N^{\prime} \cong A$, we have $N^{\prime} \cong N^{\prime} \otimes_{A}$ $A \cong N^{\prime} \otimes_{A} M \otimes_{B} N=E \otimes_{B} N$ with $E=N^{\prime} \otimes_{A} M$. Similarly, $M^{\prime} \cong$ $M \otimes_{B} F$ with $F=N \otimes_{A} M^{\prime}$. Furthermore, $E \otimes_{B} F \cong F \otimes_{B} E \cong B$. We shall show $E \cong B \otimes_{R} P$ and $F \cong B \otimes_{R} P^{-1}$ for some invertible $R$-module $P$.

Let $P=\operatorname{Hom}_{B e}(B, E)$ and note that if $p \in P$ then $p \otimes 1: B \otimes{ }_{B} F \rightarrow$ $E \otimes_{B} F$ is an element of $\operatorname{Hom}_{B^{e}}(F, B)$. Clearly the resulting map $P=\operatorname{Hom}_{B^{e}}(B, E) \rightarrow \operatorname{Hom}_{B} e(F, B)$ is an isomorphism. We set $Q=$ $\operatorname{Hom}_{B^{e}}(E, B)$ and note that $Q$ is also isomorphic to $\operatorname{Hom}_{B^{e}}(B, F)$. Also note, $\operatorname{Hom}_{B^{e}}(E, E) \cong \operatorname{Hom}_{B^{e}}(F, F) \cong \operatorname{Hom}_{B^{e}}(B, B) \cong R$. Thus, for $p \in P, q \in Q$ both compositions $p \circ q$ and $q \circ p$ are given by elements of $R$, necessarily the same element of $R$ since $(p q) p=p(q p)$. Thus, there is a well defined map $P \otimes_{R} Q \rightarrow R$ given by composition.

Let $\theta: B \otimes_{R} B \otimes_{R} \Omega \rightarrow B \otimes_{R} B$ be the separator for $B$. If we apply ()$_{B} \otimes E$ to the appropriate factors we obtain an isomorphism $\theta_{E}: B \otimes_{R} E \otimes_{R} \Omega \rightarrow E \otimes_{R} B$ and, with $\pi_{E}: E \otimes_{R} B \rightarrow E$ the module action map, a surjection $\pi_{E} \circ \theta_{E}: B \otimes_{R} E \otimes_{R} \Omega \rightarrow E$. In the $B$ variable this is a $B^{e}$-module homomorphism. It follows that $P$ is sufficiently rich that $(b \otimes p) \rightarrow p(b): B \otimes_{R} P \rightarrow E$ is surjective. Similarly, $E \otimes_{R} Q \rightarrow B$ is surjective and it follows that $P \otimes_{R} Q \rightarrow R$ is surjective. If $\sum p_{i} \otimes q_{i} \in P \otimes_{R} Q$ satisfies $\sum p_{i} q_{i}=1$ then the usual argument shows that $r \rightarrow r \sum p_{i} \otimes q_{i}$ is an inverse for $P \otimes_{R} Q \rightarrow R$. Hence, $P$ is an invertible $R$-module with inverse $Q$. It follows easily that $E \cong B \otimes_{R} P$ and $F \cong B \otimes_{R} P^{-1}$.

Now we have $N^{\prime} \cong E \otimes_{B} N \cong B \otimes_{B} N \otimes_{R} P \cong N \otimes_{R} P$ and, similarly, $M^{\prime} \cong M \otimes_{R} P^{-1}$.

If we let $Q_{B}(A)$ denote the set of equivalence classes of data presenting $A$ as a $B$-elementary algebra we have now shown that $\operatorname{Pic}(R)$ acts transitively on $Q_{B}(A)$ and that the isotropy group of a point $(N, M, \lambda)$ is the image of $\operatorname{Aut}_{R}(A)$. If we fix such a point then:

Proposition 5.5. Given Morita equivalent central separable $R$ algebras $A$ and $B$, there is an exact sequence

$$
0 \longrightarrow \operatorname{Inn}(A) \longrightarrow \operatorname{Aut}_{R}(A) \longrightarrow \operatorname{Pic}(R) \longrightarrow Q_{B}(A) \longrightarrow 0 \text {. }
$$

This is an exact analogue of the classical result (cf. [20] and [8], Ch. 2, §6).

Now Knus [15] has shown that in the classical situation of algebras with identity the image of $\operatorname{Aut}_{R}(A)$ in $\operatorname{Pic}(R)$ is contained in the torsion subgroup. Thus, if $A$ has an identity and $\operatorname{Pic}(R)$ is not a torsion group then $Q_{B}(A)$ is nontrivial. However, regardless of what $\operatorname{Pic}(R)$ is, it is easy to find a central separable $R$-algebra $A$ (without identity) for which $Q_{B}(A)=0$ for all $B$ (note $Q_{B}(A)$ is 
actually independent of $B$ ). For example, if $M$ is the direct sum of countably many copies of $R, N=\operatorname{Hom}_{R}(M, R)$ the direct product, and $\lambda: N \otimes_{R} M \rightarrow R$ the standard pairing, then the $R$-elementary algebra $A=M \otimes_{R} N$ satisfies $Q_{R}(A)=(0)$. This follows directly from the fact that $M \cong M \otimes_{R} P$ for any invertible $R$-module $P$.

It is well known that if $R=C(X)$-the algebra of continuous complex valued functions on $X$-for a compact Hausdorff space $X$, then $\operatorname{Pic}(R) \cong H^{2}(X, Z)$. The same thing is true for any commutative Banach algebra with maximal ideal space $X$ (cf. [10]). Thus, for a a commutative Banach algebra $R$ and any central separable $R$-algebra $A$ for which $Q(A)=Q_{A}(A)=(0)$, the group $\operatorname{Aut}_{R}(A) / \operatorname{Inn}(A)$ is isomorphic to the second Cech cohomology group $H^{2}(X, Z)$, where $X$ is the maximal ideal space of $R$. It was this connection with Banach algebra theory, pursued in a somewhat different way in [6], that led us to consider central separable algebras without identity.

Suppose a Morita equivalence between $A$ and $B$ is implemented by modules $M$ and $N$ so that $M \otimes_{B} N \cong A$. If $\mu: M \rightarrow M$ is a left $A$-right $B$-module endomorphism, then $\mu \otimes 1: M \otimes_{B} N \rightarrow M \otimes_{B} N$ is an $A^{e}$-module endomorphism of $A$ and, hence, given by an element of $R$. This has the following proposition as a consequence:

Proposition 5.6. Suppose $(N, M, \lambda)$ and $\left(N^{\prime}, M^{\prime}, \lambda^{\prime}\right)$ belong to the same equivalence class in $Q_{B}(A)$. Then the isomorphisms $\mu: M^{\prime} \rightarrow$ $M$ and $\nu: N^{\prime} \rightarrow N$ which determine the equivalence are unique up to the action $r \rightarrow\left(r n, r^{-1} m\right)$ of the invertible group of $R$ on $N \times M$.

6. Cohomology. The results of $\S 4$ suggest that, as with the classical Brauer group (cf. [12]), $\widetilde{B}(R)$ can be computed for certain rings $R$ by representing $R$ as the ring of sections of a sheaf of local rings for which $\widetilde{B}$ is trivial and then using sheaf cohomology methods. We shall show how this method is successful when $R=C(X)$ for $X$ a compact Hausdorff space and partially successful when $R=\mathcal{O}(X)$ for $X$ a "nice" compact subset of a Stein space. Here $\mathscr{O}(X)$ is the algebra of functions holomorphic in a neighborhood of $X$. For $R=$ $C(X)$, the result is $\widetilde{B}(R) \cong H^{3}(X, Z)$. Compare this with the classical result that $B(C(X))$ is the torsion subgroup of $H^{3}(X, Z)$ (cf. [12]). For $R=\mathscr{O}(X), X$ an appropriate compact subset of a Stein space, we get an injection $\widetilde{B}(R) \rightarrow H^{3}(X, Z)$. However, we have not been able to prove it is also a surjection.

We begin with some preliminary results concerning the algebra $\mathcal{O}(X)$. Thus, let $X$ be a compact subset of a Stein space (e.g., $X$ could be a compact subset of $C^{n}$ ) and let $\mathscr{O}(X)$ denote the algebra of functions holomorphic in a neighborhood of $X$. In other words, $\mathscr{O}(X)$ is the inductive limit of the algebras $\mathcal{O}(U)$ where $U$ ranges over open 
sets containing $X$. By a result of Harvey and Wells, if every complex homomorphism of $\mathscr{O}(X)$ is given by point evaluation at a point of $X$, then Cartan's Theorems A and B hold for coherent analytic sheaves on $X$ (cf. [14], Th. 3.3). We shall call $X$ a Stein compact set in this case. For example, if $X$ has a fundamental system of neighborhoods which are Stein spaces, then $X$ is Stein compact.

We shall need to know that $X$ has a basis for its topology consisting of sets $U$ which also satisfy Cartan's Theorem B (i.e., sets on which any coherent sheaf is acyclic). In fact, sets defined by inequalities $\left|f_{1}(x)\right|<r_{1}, \cdots,\left|f_{n}(x)\right|<r_{n}$ with $f_{1}, \cdots, f_{n} \in \mathcal{O}(X)$ have this property and do comprise a base for the topology.

Proposition 6.1. Let $X$ be a Stein compact subset of a Stein space and let $U \subset X$ have the property that every coherent analytic sheaf on $X$ is acyclic on $U$. Then $\mathscr{O}(U)$ is a flat $\mathscr{O}(X)$ module.

Proof. To prove that an $R$-module $M$ is flat it suffices to prove that $M \otimes_{R} I \rightarrow M \otimes_{R} R=M$ is injective whenever $I$ is a finitely generated ideal of $R$ (cf. [17], Ch. 2, Th. 1).

Thus, let $f_{1}, \cdots, f_{n}$ be generators of an ideal $I$ of $\mathscr{O}(X)$. Let ${ }_{x} \mathcal{O}$ denote the restriction to $X$ of the sheaf of germs of holomorphic functions in the ambient Stein space. Then $\left(g_{1}, \cdots, g_{n}\right) \rightarrow \sum f_{i} g_{i}:_{x} \mathcal{O}^{n} \rightarrow$ ${ }_{x} \mathcal{O}$ defines a homomorphism of sheaves of ${ }_{x} \mathcal{O}$ modules which we shall call $\mu$. Since $\operatorname{ker}(\mu)$ is coherent (cf. [13], Ch. 11, §13) and $X$ satisfies Cartan's Theorem A, there is a homomorphism $\nu:{ }_{x} \mathcal{O} \rightarrow{ }_{x} \mathcal{O}^{n}$ with image $\operatorname{ker}(\mu)$. Hence, we have an exact sequence

$$
0 \longrightarrow K \longrightarrow{ }_{x} \mathcal{O}^{m} \stackrel{\nu}{\longrightarrow}{ }_{x} \mathcal{O}^{n} \stackrel{\mu}{\longrightarrow}{ }_{x} \mathcal{\longrightarrow} Q \longrightarrow 0
$$

where $Q$ is the cokernel of $\mu$ and $K$ is the kernel of $\nu$ and, hence, each is a coherent sheaf.

From the fact that coherent sheaves are acyclic on $X$ and $U$ we conclude that the exactness of this sequence is preserved by passing to sections over $X$ or over $U$. Passing to sections over $X$ yields that $I=\operatorname{coker}\left\{\nu: \mathcal{O}(X)^{m} \rightarrow \mathscr{O}(X)^{n}\right\}$. This, and the exactness of the sequence for sections over $U$ and the fact that $\mathscr{O}(U) \otimes_{\mathcal{O}(X)} \mathscr{O}(X)=$ $\mathscr{O}(U)$ yields that $\mathscr{O}(U) \otimes_{\mathcal{O}(X)} I=\operatorname{coker}\left\{\nu: \mathcal{O}(U)^{m} \rightarrow \mathscr{O}(U)^{n}\right\}$ as well as the fact that this maps injectively into $\mathscr{O}(U)=\mathscr{O}(U) \otimes_{\mathcal{O}(X)} \mathcal{O}(X)$. This completes the proof.

The next proposition spells out the properties of $\mathcal{O}(X)$ and $C(X)$ which allow us to proceed with our cohomology program:

Proposition 6.2. If $R=C(X)$ for $X$ a compact Hausdorff space or $R=\mathscr{O}(X)$ for $X$ a Stein compact subset of a Stein space, then 
$R$ is the ring of global sections of a sheaf of rings $\mathscr{R}$ such that:

(1) $\mathscr{R}$ is an acyclic sheaf of Henselian local rings, each of which is algebraically closed modulo its maximal ideal;

(2) each $R$-module $M$ is isomorphic to the module of global sections of the sheaf $M \otimes_{R} \mathscr{R}$;

(3) for each $R$-module $M$ the sheaf $M \otimes_{R} \mathscr{R}$ is acyclic.

Proof. If $R=C(X)$ we choose the sheaf of germs of continuous complex valued functions for $\mathscr{R}$. If $R=\mathscr{O}(X)$ we choose $\mathscr{R}={ }_{X} O$. In either case, we fix a finite open cover of $X$ and let

(*) $\quad 0 \longrightarrow R \longrightarrow C^{0} \longrightarrow C^{1} \longrightarrow \cdots \longrightarrow C^{k} \longrightarrow 0$

be the corresponding complex of alternating Čech cochains for the sheaf $\mathscr{R}$.

In the case $R=C(X)$ this complex is exact and $R$-split (a partition of unity yields a contracting homotopy in the usual way). Now $M \otimes_{R} \mathscr{R}$ is the sheaf of germs of the presheaf $U \rightarrow M \otimes_{R} R(U)$ where $R(U)$ is the ring of sections of $\mathscr{R}$ over $U$. If we apply $M \otimes_{R}(\quad)$ to $\left(^{*}\right)$ we obtain the complex of Čech cochains for this presheaf and the given cover. Since $\left(^{*}\right)$ is $R$-split, it remains exact when tensored with $M$. On passing to the limit over refinement of open covers we conclude that $M$ is the module of global sections of $M \otimes_{R} \mathscr{R}$ (from exactness at the first two stages) and that $M \otimes_{R} \mathscr{R}$ has vanishing Čech cohomology and, hence, is acyclic.

For $R=\mathscr{O}(X)$ we proceed as above except we restrict attention to open covers with the property that each finite intersection of members of the cover is a set on which coherent sheaves are acyclic. This ensures that each $C^{p}$ is a flat $R$-module (by Prop. 6.1) and, hence, that whenever $\left({ }^{*}\right)$ is exact its tensor product with $M$ with also be exact. However, $\left(^{*}\right)$ is always exact for such a cover since $\mathscr{O}(X)=\mathscr{R}$ is acyclic on $X$ and on each finite intersection of sets in the cover. By the discussion preceding Proposition 6.1, there are arbitrarily fine covers of the required type and, hence, the argument of the previous paragraph works in this case as well.

It only remains to show that each stalk of $\mathscr{R}$ is a Henselian local ring with algebraically closed residual field. Everything but Henselian is obvious and Henselian is well known. For example, the stalks in ${ }_{x} O$ are convergent power series rings and, thus, Henselian by [18], Chapter VII, $\S 45$. Any finite subset of the ring of germs of continuous functions is contained in a subring which is a quotient of a convergent power series ring and, thus, Henselian by [18], Chapter VII, 43.4; it follows that the ring of germs of continuous functions is Henselian.

In what follows, we assume $R$ is $C(X)$ (resp. $O(X)$ ) for $X$ compact 
(resp. Stein compact) and $\mathscr{R}$ will denote the sheaf of the above proposition.

We let $\mathscr{R}^{*}$ denote the sheaf of invertible groups of the sheaf $\mathscr{R}$.

Our first objective is to construct a homomorphism $\delta: \widetilde{B}(R) \rightarrow$ $H^{2}\left(X, \mathscr{R}^{*}\right)$, where $H^{2}\left(X, \mathscr{R}^{*}\right)$ is the second sheaf cohomology group of the sheaf $\mathscr{R}^{*}$. This construction is similar to that in [9].

Let $A$ be a central separable $R$-algebra and denote the sheaf of algebras $A \otimes_{R} \mathscr{R}$ by $\mathscr{A}$. For each $x \in X$ let $\mathscr{R}_{x}$ (resp. $\mathscr{A}_{x}$ ) denote the stalk of $\mathscr{R}$ (resp. $\mathscr{A}$ ) at $x$. For each open set $U \subset X$ let $R(U)$ denote the ring of sections over $U$ of $\mathscr{R}$ and set $A(U)=A \otimes_{R} R(U)$. Then $\mathscr{A}$ is the sheaf of germs of the presheaf $U \rightarrow A(U)$. For each $U, A(U)$ is a central separable $R(U)$ algebra.

Now for each $x \in X, \mathscr{A}_{x}=\lim \{A(U): x \in U\}$ is a central separable algebra over a Henselian local ring $\mathscr{R}_{x}$ with algebraically complete residual field. Hence, $\mathscr{A}_{x}$ is an $\mathscr{R}_{x}$-elementary algebra by Proposition 4.9. This followed from the existence of a rank one idempotent in $\mathscr{A}_{x}$. It is easy to see that such an idempotent must be the germ of a rank one idempotent in $A(U)$ for some neighborhood $U$ of $x$. Thus, $A(U)$ is $R(U)$-elementary. Let $\left\{U_{i}\right\}_{i=1}^{n}$ be a finite open cover of $X$ by neighborhood for which $A(U)$ has this property. Thus, we have elementary $R\left(U_{i}\right)$ algebras $M_{i} \otimes_{R} N_{i}$ and algebra isomorphisms $\varphi_{i}: A\left(U_{i}\right) \rightarrow M_{i} \otimes_{R} N_{i}$.

If $x \in V \subset U_{i} \cap U_{j}$ then, on tensoring with $R(V)$, we have isomorphisms $A(V) \rightarrow\left(M_{i} \otimes_{R} N_{i}\right) \otimes_{R\left(J_{i}\right)} R(V)$ and $A(V) \rightarrow\left(M_{j} \otimes_{R} N_{j}\right) \otimes_{R\left(U_{i}\right)} R(V)$. In other words, we have two sets of data in $Q_{R}(A(V))$. By Proposition 5.5 one set is obtained from the other by application of an element of $\operatorname{Pic}(R(V))$. Since $\operatorname{Pic}\left(\mathscr{R}_{x}\right)=(0)$ and $\operatorname{Pic}\left(\mathscr{R}_{x}\right)=\lim \{\operatorname{Pic}(R(V)): x \in V\}$, we conclude that for sufficiently small $V$ this element of $\operatorname{Pic}(R(V))$ must be zero. Then the two sets of data belong to the same element of $Q_{R}(A)$. It follows that if we replace our original cover by a sufficiently fine refinement we may obtain the following: if $U_{i} \cap U_{j}=U_{i j}$ and $M_{i}(V)=M_{i} \otimes_{R(U i)} R(V), N_{i}(V)=N_{i} \otimes_{R(U i)} R(V)$ there are $R$-module homomorphisms $\mu_{i j}: M_{j}\left(U_{i j}\right) \rightarrow M_{i}\left(U_{i j}\right)$ and $\nu_{i j}: N_{j}\left(U_{i j}\right) \rightarrow N_{i}\left(U_{i j}\right)$ such that $\varphi_{i} \circ \varphi_{j}^{-1}=\mu_{i j} \otimes \nu_{i j}: M_{j}\left(U_{i j}\right) \otimes_{R\left(U_{i j}\right)} N_{j}\left(U_{i j}\right) \rightarrow M_{i}\left(U_{i j}\right) \otimes_{R\left(U_{i j}\right)} N_{i}\left(U_{i j}\right)$. We next set $U_{i j k}=U_{i} \cap U_{j} \cap U_{k}$ and consider the maps $\mu_{i j} \circ \mu_{j k} \circ \mu_{k i}: M_{i}\left(U_{i j k}\right) \rightarrow$ $M_{i}\left(U_{i j k}\right)$ and $\nu_{i j} \circ \nu_{j k} \circ \nu_{k i}: N_{i}\left(U_{i j k}\right) \rightarrow N_{i}\left(U_{i j k}\right)$. By Proposition 5.6 these are given by miltiplication by elements $r_{i j k}$ and $r_{i j k}^{-1}$, respectively, in $R\left(U_{i j k}\right)$. If we are careful to choose $\mu_{i j}$ and $\nu_{i j}$ so that $\mu_{j i}=\mu_{i j}^{-1}$ and $\nu_{j i}=\nu_{j i}^{-1}$, then a simple computation shows that $\left\{r_{i j k}\right\}$ form a 2cocycle for the cover $\left\{U_{i}\right\}$ and the sheaf $\mathscr{R}^{*}$. On passing to the limit over refinements, we obtain an element $\delta(A) \in H^{2}\left(X, \mathscr{R}^{*}\right)$.

The element $\delta(A)$ does not depend on the choice of the isomorphisms $\varphi_{i}: A\left(U_{i}\right) \rightarrow M_{i} \otimes_{R\left(U_{i}\right)} N_{i}$. In fact, suppose $\varphi_{i}^{\prime}: A\left(U_{i}\right) \rightarrow$ 
$M_{1}^{\prime} \otimes_{R\left(U_{i}\right)} N_{i}^{\prime}$ is another choice. Then by passing to a refinement of the cover if necessary, we may assume $\varphi_{i}^{\prime} \circ \varphi_{i}^{-1}=\mu_{i} \otimes \nu_{i}: M_{i} \otimes_{R\left(U_{i}\right)} N_{i} \rightarrow$ $M_{i}^{\prime} \otimes_{R\left(U_{i}\right)} N_{1}^{\prime}$ for $R\left(U_{i}\right)$ module isomorphisms $\mu_{i}$ and $\nu_{i}$. We then conclude from Proposition 5.6 that $\mu_{i j}^{\prime}=r_{i j} \mu_{i} \mu_{i j} \mu_{j}^{-1}$ for $r_{i j} \in R\left(U_{i j}\right)$ and, hence, that $r_{i j k}^{i}=r_{i j} r_{j k} r_{k i} r_{i j k}$ where primes indicate the data constructed from the $\varphi_{i}^{\prime \prime}$ s. In other words, $\left\{r_{i j_{k}}^{\prime}\right\}$ differs from $\left\{r_{i j k}\right\}$ by a coboundary and, hence, determines the same class in $H^{2}\left(X, \mathscr{R}^{*}\right)$.

Now if $A$ is Morita equivalent to another algebra $B$, say $B=$ $M \otimes_{A} N$ and $A=N \otimes_{B} M$, then the data $\left\{\left(M_{i}, N_{i}\right)\right\}$ for $A$, yield data $M \otimes_{A} M_{i}$ and $N_{i} \otimes_{A} N$ for $B$ which leads to the same cocycle $\left\{r_{i j k}\right\}$. Hence, we have a well defined map $\delta: \widetilde{B}(R) \rightarrow H^{2}\left(X, \mathscr{R}^{*}\right)$. It is easy to see that it is a group homomorphism.

We would like to show that $\delta$ is injective. That is, if $\delta(A)=0$ we would like to show that $A \cong M \otimes_{R} N$ for modules $M$ and $N$ with a map $\lambda: N \otimes_{R} M \rightarrow R$ which defines the multiplication in $A$. This is easy enough to do at the sheaf level:

Proposition 6.3. If $\delta(A)=0$ there are sheaves $\mathscr{N}$ and $\mathscr{M}$ of $\mathscr{R}$-modules and an $\mathscr{R}$-module homomorphism $\lambda: \mathscr{N} \otimes_{\mathscr{A}} \mathscr{M} \rightarrow \mathscr{R}$ such that $\mathscr{A} \cong \mathscr{M} \otimes \mathscr{N}$ as sheaves of $\mathscr{R}$-modules in such a way that the multiplication map $\mathscr{A} \otimes, \mathscr{A} \rightarrow \mathscr{A}$ is equivalent to $1 \otimes$ $\lambda \otimes 1: \mathscr{H} \otimes_{\mathbb{A}} \mathscr{N} \otimes_{\mathbb{R}} \mathscr{M} \otimes_{\pi} \mathscr{N} \rightarrow \mathscr{M} \otimes_{\mathscr{A}} \mathscr{N} . \quad$ Furthermore, the sheaves $\mathscr{M}$ and $\mathscr{N}$ can be chosen so that they are locally $\mathscr{R}$-module direct summands of $\mathscr{A}$.

Proof. Suppose we have an open cover $\left\{U_{i}\right\}$ of $X$, data $\left(N_{i}, M_{i}, \lambda_{i}\right)$ for elementary $R\left(U_{i}\right)$ algebras, isomorphisms $\varphi_{i}: A\left(U_{i}\right) \rightarrow M_{i} \otimes_{R\left(U_{i}\right)} N_{i}$ and maps $\mu_{i j}: M_{j}\left(U_{i j}\right) \rightarrow M_{i}\left(U_{i j}\right), \nu_{i j}: N_{j}\left(U_{i j}\right) \rightarrow N_{i}\left(U_{i j}\right)$ with $\lambda_{i}\left(\mu_{i j} m \otimes\right.$ $\left.\nu_{i j} n\right)=\lambda_{j}(m \otimes n)$. Suppose $\left\{r_{i j k}\right\}$ is constructed as above, so that $r_{i j k}=\mu_{i j} \circ \mu_{j k} \circ \mu_{k i}$ and $r_{i j k}^{-1}=\nu_{i j} \circ \nu_{j k} \circ \nu_{k i}$. If $\left\{r_{i j k}\right\}$ determines the zero class in $H^{2}\left(X, \mathscr{R}^{*}\right)$, then by passing to a refinement if necessary, we may assume $r_{i j k}=r_{i j} r_{j k} r_{k l}$ for an alternating Čech 1 cochain $\left\{r_{i j}\right\}$ for the cover $\left\{U_{i}\right\}$ and the sheaf $\mathscr{R}^{*}$. If we set $\mu_{i j}^{\prime}=r_{i j}^{-1} \mu_{i j}$ and $\nu_{i j}^{\prime}=r_{i j} \nu_{i j}$, then $\mu_{i j}^{\prime} \circ \mu_{2 k}^{\prime} \circ \mu_{k i}^{\prime}=1=\nu_{i j}^{\prime} \circ \nu_{j k}^{\prime} \circ \nu_{k i}^{\prime}$. This allows us to construct sheaves of $\mathscr{R}$-modules $\mathscr{C}$ and $\mathscr{N}$ on $X$ as follows: on $U_{i}$, $\mathscr{C l}$ is $M_{i} \otimes_{R\left(U_{i}\right)} \mathscr{R}$ where, over points of $U_{i} \cap U_{j}$ we identify elements of $M_{i} \otimes_{R\left(J_{i}\right)} \mathscr{R}$ with elements of $M_{j} \otimes_{R\left(U_{j}\right)}$. $\mathscr{N}$ is constructed in a similar fashion.

The consistency requirement for $\mu_{i j}, \nu_{i j}$ and $\lambda_{i}$ and $\lambda_{j}$ implies that the $\lambda_{i}: N_{i} \otimes_{R\left(U_{i}\right)} M_{i} \rightarrow R\left(U_{i}\right)$ fit together to define a global sheaf homomorphism $\lambda: \mathscr{N} \otimes_{R} \mathscr{M} \rightarrow \mathscr{R}$. Furthermore, the maps $\varphi_{i}: A\left(U_{i}\right) \rightarrow$ $M_{i} \otimes_{R\left(U_{i}\right)} N_{i}$ fit together to define an isomorphism $\varphi: \mathscr{A} \rightarrow \mathscr{M} \otimes_{R} \mathscr{N}$ of sheaves of algebras, where $\lambda$ determines the multiplication on $\mathscr{L} \otimes_{R} \mathscr{N}^{\prime}$. 
That $\mathscr{M}$ and $\mathscr{N}$ can locally be realized as $\mathscr{R}$-module direct summands of $\mathscr{A}$ follows from the fact that $M_{i}$ and $N_{i}$ can be chosen of the form $A p_{i}$ and $p_{i} A$ for a rank one idempotent $p_{i} \in\left(A\left(U_{i}\right)\right)$ provided the cover is sufficiently fine.

The problem of showing that $\delta$ is injective now boils down to this: with $\mathscr{M}$ and $\mathscr{N}$ as above, is $\Gamma\left(X, \mathscr{M} \otimes_{R} \mathscr{N}\right) \cong \Gamma(X, \mathscr{M}) \otimes_{R}$ $\Gamma(X, \mathscr{N})$ ? If this is true then $A$ is isomorphic to the elementary algebra determined by $(N, M, \lambda)$ where $M=\Gamma(X, \mathscr{C})$ and $N=$ $\Gamma(X, \mathscr{N})$, since Proposition 6.2 implies $A \cong \Gamma(X, \mathscr{A})$.

For $R=C(X)$ there is no problem. In this case, $\mathscr{R}$, the sheaf of germs of continuous functions on $X$, is a fine sheaf. Hence, any sheaf of $\mathscr{R}$-modules is a soft sheaf. This means that sections over closed sets are restrictions of global sections and it implies that the sheaf is acyclic (cf. [11]). It follows that there are free $\mathscr{R}$ modules $\mathscr{F}_{1}$ and $\mathscr{F}_{0}$ and an exact sequence of $\mathscr{R}$-modules $\mathscr{F}_{1} \rightarrow \mathscr{F}_{0} \rightarrow$ $\mathscr{N} \rightarrow 0$. This yields an exact sequence $\mathscr{M} \otimes_{R} \mathscr{F}_{1} \rightarrow \mathscr{M} \otimes_{R} \mathscr{F}_{0} \rightarrow$ $\mathscr{M} \otimes_{R} \mathscr{N} \rightarrow 0$ which remains exact when we pass to global sections because sheaves of $\mathscr{R}$-modules are acyclic. However, if $F_{i}=$ $\Gamma\left(X, \mathscr{F}_{i}\right)$ for $i=0,1$, then $\Gamma\left(X, \mathscr{M} \otimes_{R} \mathscr{F}_{i}\right)=M \otimes F_{i}$. Hence, we have an exact sequence $M \otimes_{R} F_{1} \rightarrow M \otimes_{R} F_{0} \rightarrow \Gamma\left(X, \mathscr{M} \otimes_{R} \mathscr{N}\right) \rightarrow 0$. Since the cokernel of $M \otimes_{R} F_{1} \rightarrow M \otimes_{R} F_{0}$ is $M \otimes_{R} N$, we have proved that $\Gamma\left(X, \mathscr{M} \otimes_{R} \mathscr{N}\right) \cong M \otimes_{R} N$ when $R=C(X)$.

The case $R=\mathscr{O}(X)$ raises a fundamental difficulty. First, a positive result: suppose that $A$ is a finitely presented $\mathscr{O}(X)=R$ module. Then $\mathscr{A}=\mathscr{A} \otimes_{R} \mathscr{R}$ is a finitely presented sheaf of $\mathscr{R}$ modules and, hence, $\mathscr{A}$ is a coherent analytic sheaf on a neighborhood of $X$. Since $\mathscr{C}$ and $\mathscr{N}$ are locally direct summands of $\mathscr{A}$ (Prop. 6.3) they too are coherent analytic sheaves in a neighborhood of $X$. It follows from Cartan's Theorem A, that there is an exact sequence ${ }_{x} \mathcal{O}^{m} \rightarrow{ }_{x} \mathcal{O}^{n} \rightarrow \mathscr{N}$ of sheaves of ${ }_{x} \mathcal{O}$ modules. Since coherent sheaves are acyclic by Cartan's Theorem B, the argument of the above paragraph goes through and we again conclude that $\Gamma\left(X, \mathscr{M} \otimes_{R} \mathscr{N}\right) \cong M \otimes_{R} N$.

To be able to conclude from this that $\delta$ is injective we need to know that every class in $\widetilde{B}(\mathcal{O}(X))$ contains an algebra $A$ which is finitely presented over $\mathscr{O}(X)$. Now if $\mathscr{O}(X)$ is noetherian, then Corollary 4.2 implies that each class contains an algebra $A$ which is a finitely generated $R$-module. Then, applying noetherian again, we conclude that $A$ is finitely presented. Thus, we have proved:

Proposition 6.4. Under the hypotheses of Proposition 6.2, $\delta(A)=$ 0 implies $[A]=0$ in $\widetilde{B}(R)$ under each of the following conditions:

(1) $R=C(X)$

(2) $R=\mathscr{O}(X)$ and $\mathscr{O}(X)$ is noetherian; 


\section{(3) $A$ is a finitely presented $R$-module.}

The algebra $\mathcal{O}(X)$ need not be noetherian. For example, let $X$ be a convergent sequence of points in $C$. There are more subtle examples, with $X$ connected, involving sets $X \subset C^{n}$ for which there is a subvariety which meets the boundary of $X$ in a nonlocally connected set. However, if $X$ is a holomorphically convex subset of a domain of holomorphy which is semi-analytic (defined by finitely many analytic inequalities) then $\mathscr{O}(X)$ is noetherian (cf. [23]).

Proposition 6.4 suggests the following definition:

Definition 6.1. For any commutative ring $R$ let $\bar{B}(R)$ denote the subgroup of $\widetilde{B}(R)$ consisting of classes which contain a finitely presented algebra.

We know that $\widetilde{B}(R)=\bar{B}(R)$ for $R$ noetherian. We can't prove that $\widetilde{B}(R)=\bar{B}(R)$ in general, and we suspect that it is not true. If they are not equal in general, then Proposition 6.4 and what follows suggest that $\bar{B}$ may be the more appropriate functor in some circumstances.

Proposition 6.5. If $R=\operatorname{Lim} R_{\alpha}$ for a direct limit system of rings $\left\{R_{\alpha}\right\}$, then $\bar{B}(R)=\underset{\longrightarrow}{\lim } \bar{B}\left(R_{\alpha}\right)$.

Proof. Let $A$ be a finitely presented central separable $R$-algebra. Then, as an $R$-module, $A$ is the cokernel of a map $R^{n} \rightarrow R^{m}$, i.e., a matrix with entries from $R$. By lifting each of these entries to some $R_{\alpha}$ we obtain a map $R_{\alpha}^{n} \rightarrow R_{\alpha}^{m}$ having as cokernel an $R$-module $A_{\alpha}$ such that $A_{\alpha} \otimes_{R_{\alpha}} R=A$. Now the multiplication map for $A$ and each of the homomorphisms $A \otimes_{R} A \rightarrow A \otimes_{R} A$ belonging to $\Omega(A)$ may also be represented by finite matrices over $R$. The associative law and conditions for membership in $\Omega(A)$ may each be expressed in terms of finitely many polynomial equations in the entries of these matrices. Thus, for large enough $\alpha, A$ lifts to an associative $R_{\alpha}$ algebra $A_{\alpha}$ and $\Omega(A)$ lifts to a submodule $\Omega_{\alpha} \subset \Omega\left(A_{\alpha}\right)$. Now the surjective map tr: $A \otimes_{R} \Omega(A) \rightarrow R$ may also be represented by a finite matrix. It follows that for large $A$, the restriction of

$$
\operatorname{tr}: A_{\alpha} \otimes_{R_{\alpha}} \Omega\left(A_{\alpha}\right) \longrightarrow Z\left(A_{\alpha}\right)
$$

to $A_{\alpha} \otimes_{R_{\alpha}} \Omega_{\alpha}$ has range exactly $R$ (not a quotient of $R$ ). Thus, $A_{\alpha}$ is a faithful $R$-module and Proposition 3.8 implies that $A_{\alpha}$ is central separable. Thus, we have proved that $\lim \bar{B}\left(R_{\alpha}\right) \rightarrow \bar{B}(R)$ is surjective.

Now suppose $A_{\alpha}$ is central separable over $R_{\alpha}$ and finitely presented. If $A=A_{\alpha} \otimes_{R_{\alpha}} R$ is $R$-elementary, say $A \cong M \otimes_{R} N$, then 
each of $M$ and $N$ is finitely presented. Thus, they lift to $R_{\alpha}$-modules $M_{\alpha}$ and $N_{\alpha}$ for large $\alpha$. The surjection $\lambda: N \otimes_{R} M$ lifts to a surjection $\lambda_{\alpha}: N_{\alpha} \otimes_{R_{\alpha}} M_{\alpha} \rightarrow R_{\alpha}$ and the isomorphism $A \rightarrow M \otimes_{R} N$ lifts to an isomorphism $A_{\alpha} \rightarrow M_{\alpha} \otimes_{R_{\alpha}} N_{\alpha}$ for large enough $\alpha$. Thus $\lim \bar{B}\left(R_{\alpha}\right) \rightarrow$ $\bar{B}(R)$ is injective as well.

Since every ring is a direct limit of noetherian rings, the only way the above proposition could remain true with $\bar{B}$ replaced by $\widetilde{B}$ is if $\bar{B}=\widetilde{B}$.

We now return to the situation where $R=C(X)$ or $\mathscr{O}(X)$ and continue our discussion of the map $\delta$.

Proposition 6.6. If $R=C(X)$ for $X$ compact, then $\delta: \widetilde{B}(R) \rightarrow$ $H^{2}\left(X, \mathscr{R}^{*}\right)$ is surjective.

Proof. We closely follow Dixmier-Douady [9]. The method is to use an element of $H^{2}\left(X, R^{*}\right)$ to construct a locally trivial bundle of elementary $R$-algebras; the algebra of sections then yields an element of $\widetilde{B}(R)$. Up to a point the proof works as well for $\mathcal{O}(X), X$ Stein compact, as it does for $C(X)$. We shall indicate this as we go along and then point out where the problem lies that stops us from concluding $\delta$ is surjective when $R=O(X)$.

Let $H$ be separable Hilbert space and let $G$ be the group of invertible bounded linear operators on $H$. We give $G$ the operator norm topology. Let $B$ be the algebra of finite rank operators on $H$. Then $B$ is $C$-elementary; in fact, $B \cong H \otimes_{c} H^{\prime}$ where $H^{\prime}$ is the Banach space dual of $H$ and multiplication is given by the standard pairing $H^{\prime} \otimes_{c} H \rightarrow C$. The group $G$ acts as a group of automorphisms of $B$, where $g \in G$ determines the automorphism $b \rightarrow g b g^{-1}$. If $g^{\prime}: H^{\prime} \rightarrow H^{\prime}$ is the Banach space adjoint of $g$ and $g^{*}=\left(g^{\prime}\right)^{-1}$ the contragradient operator, then this automorphism can be written as $g \otimes g^{*}: H \otimes H^{\prime} \rightarrow$ $H \otimes H^{\prime}$. If $P$ is the group of all auch automorphisms, then we have an exact sequence

$$
0 \longrightarrow C^{*} \longrightarrow G \longrightarrow P \longrightarrow 0
$$

with $C^{*}$ regarded as the group of invertible scalar operators. This sequence, expresses $G$ as a locally trivial fiber bundle with base $P$ and fiber $C^{*}$. This is true not only topologically but also analytically. That is, $C^{*}, G$, and $P$ are complex Banach Lie groups in the sense of [19] and $G$ is a locally trivial holomorphic bundle over $P$.

We denote the sheaf of germs of continuous functions with values in $C^{*}, G$, and $P$ respectively by $\mathscr{C}^{*}, \mathscr{G}$, and $\mathscr{P}$. The corresponding sheaves of germs of holomorphic functions will be denoted $\mathcal{O}^{*}, \mathscr{G}_{a}, \mathscr{P}_{a}$. 
As in $\S 26$ of [9], the exact sequence $0 \rightarrow \mathscr{C}^{*} \rightarrow \mathscr{G} \rightarrow \mathscr{P} \rightarrow 0$ induces a homomorphism $k: H^{1}(X, \mathscr{P}) \rightarrow H^{2}\left(X, \mathscr{C}^{*}\right)$. Similarly, the sequence $0 \rightarrow \mathcal{O}^{*} \rightarrow G_{a} \rightarrow \mathscr{P}_{a} \rightarrow 0$ induces a homomorphism

$$
k_{a}: H^{1}\left(X, \mathscr{P}_{a}\right) \longrightarrow H^{2}\left(X, \mathscr{O}^{*}\right) \text {. }
$$

We claim that $k$ and $k_{a}$ are both isomorphisms. For $k$ this follows from Lemmas 4 and 22 of [9] and the fact that $G$ is contractable (cf. [16]). To prove it for $k_{a}$, we let $X$ be a Stein compact set and consider the commutative diagram

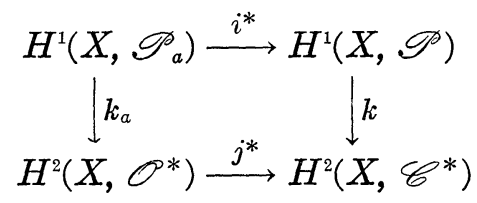

where $i^{*}$ and $j^{*}$ are induced by the inclusion maps $i: \mathscr{P}_{a} \rightarrow \mathscr{P}$ and $j: \mathscr{O}^{*} \rightarrow \mathscr{C}^{*}$. We will have that $k_{a}$ is an isomorphism if we can prove that $i^{*}$ and $j^{*}$ are isomorphisms. That $j^{*}$ is an isomorphism is a standard fact. It follows from the commutative diagram

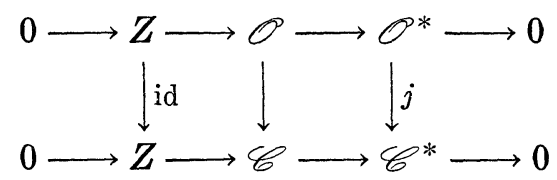

on passing to the long exact sequences of cohomology and using the fact that $\mathcal{O}$ and $\mathscr{C}$ are acyclic. Here, $Z$ is the constant sheaf of integers and $e(f)=\exp (2 \pi i f)$ for $f$ a continuous or holomorphic germ. This argument actually proves that $H^{2}\left(X, \mathcal{O}^{*}\right) \cong H^{2}\left(X, \mathscr{C}^{*}\right) \cong H^{3}(X, Z)$. That $i^{*}$ is an isomorphism follows from Theorems A and B of [19]. This is Raeburn's generalization to infinite dimensional bundles of Grauert's deep work on holomorphic fiber bundles. Although Raeburn's theorems assume the underlying space is a Stein space, the proofs work just as well for a compact Stein set. Thus, $i^{*}$ is an isomorphism and $k_{a}$ is an isomorphism.

We complete the proof that $\delta$ is surjective (for $R=C(X)$ ) by defining a map $m: H^{1}(X, \mathscr{P}) \rightarrow \widetilde{B}(C(X))$ for which the diagram

$(*)$

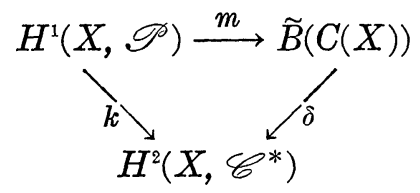

is commutative. Since $k$ is surjective, this will prove that $\delta$ is surjective.

To construct the map $m$, let an element of $H^{1}(X, \mathscr{P})$ be repre- 
sented by a Čech cocyle $\left\{\sigma_{i j}\right\}$ for $\mathscr{P}$ and the cover $\left\{U_{i}\right\}$ of $X$. By passing to a refinement, if necessary, we may assume each $\sigma_{i j}$ is the image of a section $g_{i j}$ of $\mathscr{G}$ over $U_{i} \cap U_{j}$. For each open set $U$ we let $H(U)$ and $H^{\prime}(U)$ be the $C(U)$ modules of continuous $H$ and $H^{\prime}$ valued functions, respectively, and set $B(U)=H(U) \otimes_{C(U)} H^{\prime}(U)$. The standard pairing $\lambda_{U}: H^{\prime}(U) \otimes_{C(U)} H(U) \rightarrow C(U)$ makes $B(U)$ an elementary $C(U)$ algebra. At this point we have a presheaf $U \rightarrow$ $B(U)$ of elementary algebras. We let $\mathscr{B}$ be the corresponding sheaf of germs of elementary $\mathscr{C}$ algebras. We then define a new sheaf $\mathscr{A}$ by letting $\mathscr{A}_{U_{i}}=\left.\mathscr{B}\right|_{U_{i}}$ and identifying $\mathscr{A}_{U_{i}}$ and $\mathscr{A}_{U_{j}}$ over $U_{i} \cap U_{j}$ via the automorphism $\sigma_{i j}=g_{i j} \otimes g_{i j}^{*}$ considered as maps from $\left.\mathscr{A}_{U i}\right|_{U_{i} \cap U_{j}}$ to $\left.\mathscr{A}_{U_{i}}\right|_{U_{i} \cap U_{j}}$. We then let $A$ be the algebra of global sections of the sheaf $\mathscr{A}$.

Now for each $U$ we have a map $\omega: B(U) \otimes_{c(U)} B(U) \rightarrow$ $B(U) \otimes_{C(U)} B(U)$ which is a generator for $\Omega(B(U))$ and is defined as follows: $\quad \omega\left(h_{1} \otimes h_{1}^{\prime} \otimes h_{2} \otimes h_{2}^{\prime}\right)=h_{1} \otimes h_{2}^{\prime} \otimes h_{2} \otimes h_{1}^{\prime}$ with $B(U)$ identified with $H(U) \otimes_{C(U)} H^{\prime}(U)$. Clearly $\omega$ commutes with each of the automorphisms $\sigma_{i j}=g_{i j} \otimes g_{i j}^{*}$ and, hence, defines a sheaf map $\mathscr{A} \otimes_{c} \mathscr{A} \rightarrow$ $\mathscr{A} \rightarrow \mathscr{A} \otimes_{\mathscr{G}} \mathscr{A}$. Since $\Gamma\left(\mathscr{A} \otimes_{\mathscr{B}} \mathscr{A}, X\right)=A \otimes_{c(X)} A$ (see the discussion following Proposition 6.3) this sheaf map defines an element $\omega: A \otimes_{C(X)} A \rightarrow A \otimes_{C(X)} A$ of $\Omega(A)$. The trace $\operatorname{tr}_{\omega}: A \rightarrow C(X)$ corresponding to this element is locally given by

$$
\operatorname{tr}_{\omega}\left(h \otimes h^{\prime}\right)(x)=\lambda\left(h^{\prime} \otimes h\right)(x)=h^{\prime}(x)(h(x)) .
$$

As a map from the sheaf $\mathscr{A}$ to $\mathscr{C}$ this is clearly surjective. That it is surjective on sections follows from the fact that $\mathscr{C}$ is a fine sheaf and we are dealing with sheaves of $\mathscr{C}$ modules.

It now follows from Proposition 3.8 that $A$ is a central separable $C(X)$ algebra. The correspondence $\left\{\sigma_{i j}\right\} \rightarrow A$ defines our map $m: H^{1}(X, \mathscr{P}) \rightarrow \widetilde{B}(C(X))$. It is clear from the construction of $m, \delta$ and $k$ that the diagram $\left(^{*}\right)$ is commutative. This completes the proof.

What goes wrong when $R=\mathscr{O}(X)$ ? If in constructing $m$ we begin with an analytic cocycle $\left\{\sigma_{i j}\right\}$ and use holomorphic functions and sections in the above construction we end up with two problems: is $\Gamma\left(\mathscr{A} \otimes_{\gamma_{(X)}} \mathscr{A}\right) \cong A \otimes_{O(X)} A$ where $A=\Gamma(\mathscr{A}, X)$ and $\mathscr{A}$ is the analytic sheaf of algebras constructed from $\left\{\sigma_{i j}\right\}$ ? If so, is $\operatorname{tr}_{\omega}: A \rightarrow$ $\mathcal{O}(X)$ surjective? Here we are no longer dealing with sheaves of modules over a fine sheaf. Furthermore, due to the infinite dimensionality of $H, \mathscr{A}$ fails to be a coherent analytic sheaf. A priori, we do not know that $\mathscr{A}$ has any sections other than the zero section. It may be that $A=(0)$. Now $\mathscr{A}$ is locally trivial and if it were the sheaf of sections of a bundle of Banach spaces we could apply results of Bungart [5] to get a sufficient supply of sections. However, 
the fiber of our bundle is $H \otimes H^{\prime}$ which is not complete. We could pass to a completion, but this would force us to use a completed tensor product rather than $A \otimes_{O_{(X)}} A$ in order to get $\omega$ defined. Thus, we would not be defining an element of $\widetilde{B}(\mathcal{O}(X))$. We shall discuss the relevance of this question to commutative Banach algebra theory in the next section.

In view of the isomorphisms $H^{2}\left(X, \mathscr{O}^{*}\right) \cong H^{3}(X, Z)$ and $H^{2}\left(X, \mathscr{C}^{*}\right) \cong$ $H^{3}(X, Z)$, we have the following summary of the results of this section:

THEOREM 6.7. If $R=C(X)$ for a compact Hausdorff space $X$ then $\widetilde{B}(R) \cong H^{3}(X, Z)$. If $R=\mathscr{O}(X)$ for $X$ a Stein compact set and $\mathcal{O}(X)$ is noetherian, then $\widetilde{B}(R) \cong \bar{B}(R)$ is isomorphic to a subgroup of $H^{3}(X, Z)$ containing the torsion subgroup.

The last statement of the theorem is due to the inclusion $B(R) \subset$ $\widetilde{B}(R)$ and the fact that for $R=\mathcal{O}(X), B(R)$ is isomorphic to the torsion subgroup of $H^{3}(X, Z)$ (cf. [12]).

7. Commutative Banach algebras. Let $R$ be a commutative Banach algebra with identity. The maximal ideal space $\Delta_{R}$ is a compact Hausdorff space under the weak-* topology it inherits from being a subset of the Banach space dual of $R$. If $R=C(X)$ then $\Delta_{R}=X$.

It is natural to try to characterize the Čech cohomology groups of $\Delta_{R}$ in terms of algebraic properties of the algebra $R$. In low dimension there are such characterization: $H^{0}\left(X_{R}, Z\right)$ is the additive group generated by the idempotents of $R[22], H^{1}\left(\Delta_{R}, Z\right)$ is the invertible group of $R$-modulo the image of the exponential map ([1], [21]), and $H^{2}\left(\Delta_{R}, Z\right)$ is the Picard group of $R$ [10]. For a discussion of these results and others involving various brands of $K$-theory see [24].

The Picard group is a functor defined for any commutative ring $R$ which happens to yield $H^{2}\left(\Delta_{R}, Z\right)$ when $R$ is a Banach algebra. When we began this paper we believed that $\bar{B}$ had these properties with $H^{3}$ replacing $H^{2}$. However, the difficulties encountered in $\S 6$ for $R=\mathscr{O}(X)$ now shed considerable doubt on this conjecture. If we had been able to prove $\delta$ surjective for $R=\mathscr{O}(X)$ the argument that $\bar{B}(R) \cong H^{3}\left(A_{R}, Z\right)$ would have gone as follows: The holomorphic functional calculus can be used to prove that every commutative Banach algebra $R$ with identity is the direct limit of a system $\left\{\mathscr{O}\left(X_{\alpha}\right)\right\}$, where the $X_{\alpha}$ are Stein compact sets for which $\mathcal{O}\left(X_{\alpha}\right)$ is noetherian. This can be done in such a way that $A_{R}$ is the inverse limit of the spaces $X_{\alpha}$. If we had $\bar{B}\left(\mathscr{O}\left(X_{\alpha}\right)\right) \cong H^{3}\left(X_{\alpha}, Z\right)$ we could pass to the 
limit, use the continuity of $\bar{B}$ and $H^{3}$ and conclude $\bar{B}(R) \cong H^{3}\left(\Delta_{R}, Z\right)$.

Whether or not such a program can succeed awaits settlement of the question of the surjectivity of $\delta$ for $R=\mathcal{O}(X)$. We suspect the answer is negative. In this case, a decent characterization of $H^{3}\left(\Delta_{R}, Z\right)$ may require abandoning the purely algebraic functors $\widetilde{B}$ and $\bar{B}$ and instead dealing with a Brauer group defined only for Banach or topological algebras $R$. Such a Brauer group can probably be developed using a notion of central separable algebra similar to ours but involving completed tensor products. A class of algebras of this sort was defined and studied in [6] but the idea of using them to define a Brauer group was not pursued.

\section{REFERENCES}

1. R. Arens, The group of invertible elements of a commutative Banach algebra, Studia Math., (1963), 21-23.

2. M. Auslander and O. Goldman, The Brauer group of a commutative ring, Trans. Amer. Math. Soc., 97 (1960), 367-409.

3. G. Azumaya, On maximally central algebras, Nagoya Math. J., 2 (1951), 119-150.

4. H. Bass, Algebraic K-theory, Benjamin, New York, 1968.

5. L. Bungart, On analytic fiber bundles-I, holomorphic fiber bundles with infinite dimensional fibers, Topology, 7 (1968), 55-68.

6. I. Craw, I. Raeburn and J. L. Taylor, Automorphisms of Banach Azumaya algebras, Amer. J. Math., to appear.

7. I. Craw and S. Ross, Projective separable algebras over a commutative Banach algebra.

8. F. Demeyer and E. Ingraham, Separable algebras over commutative rings, SpringerVerlag Lectures Notes in Mathematics, 181 (1971).

9. J. Dixmier and A. Douady, Champs continus d'espaces Hilbertiens et $C^{*}$-algebres, Bull. Soc. Math. France, 91 (1963), 227-284.

10. O. Forster, Functiontheoretische Hilfsmittel im der theorie der kommutativen Banach algebren, Uber. Deutsch, Math.-Verein, 76 (1974), 1-17.

11. R. Godement, Topologie algebrique et theorie des faisceaux, Hermann, Paris, 1958.

12. A. Grothendieck, Le group de Brauer I: algebras d'Azumaya et interpretations diverses, Seminaires Bourbaki, Expose, 290 (1964/65).

13. R. C. Gunning and H. Rossi, Analytic Functions of Several Complex Variables, Prentice Hall, Englewood Cliffs, N. J., 1965.

14. R. Harvey and R. O. Wells, Compact holomorphically convex subsets of a Stein manifold, Trans. Amer. Math. Soc., 136 (1969), 509-516.

15. M. A. Knus, Algebres d'Azumaya et modules projectifs, Comm. Helv. Math., 45 (1970), 372-383.

16. N. H. Kuiper, The homotopy type of the unitary group of Hilbert space, Topology, 3 (1965), 19-30.

17. H. Matsumara, Commutative Algebra, Benjamin, New York, 1970.

18. M. Nagata, Local Rings, Interscience, New York, 1962.

19. I. Raeburn, The relationship between a commutative Banach algebra and its maximal ideal space, J. Functional Anal., 25 (1977), 366-390.

20. A. Rosenberg and D. Zelinsky, Automorphisms of separable algebras, Pacific J. Math., 11 (1961), 1109-1117.

21. H. L. Royden, Function algebras, Bull. Amer. Math. Soc., 69 (1963), 281-298.

22. G. E. Shilov, On decomposition of a commutative normed ring in a direct sum 
of ideals, Math. Sb., 32 (1953), 353-364; Amer. Math. Soc. Transl., (2) 1 (1955), 37-48. 23. Y. T. Siu, Noetherianess of rings of holomorphic functions on Stein compact subsets, Proc. Amer. Math. Soc., 21 (1969), 483-489.

24. J. L. Taylor, Topological invariants of the maximal ideal space of Banach algebra, Adv. in Math., 19 (1976), 149-209.

25. - Twisted products of Banach algebras and 3rd Čech cohomology, in Ktheory and operator algebras, Springer-Verlag Lecture Notes in Mathematics, 575 (1977), 157-174.

Received December 8, 1980. Author's research supported by NSF Grant No. MCS79-02747-A01.

UNIVERSITY OF UTAH

Salt LaKe City, UT 84112 



\section{PACIFIC JOURNAL OF MATHEMATICS}

\section{EDITORS}

DONALD BABBITT (Managing Editor)

University of California

Los Angeles, California 90024

Hugo RossI

University of Utah

Salt Lake City, UT 84112

C. C. Moore and Arthur Agus

University of California

Berkeley, CA 94720
J. DugundJI

Department of Mathematics University of Southern California Los Angeles, California 90007

R. FInN and J. Milgram Stanford University Stanford, California 94305

ASSOCIATE EDITORS
R. ARNES
E. F. BeCKenbach
B. H. NeumanN
F. WOLF
K. YoSHIDA

\section{SUPPORTING INSTITUTIONS}

UNIVERSITY OF ARIZONA

UNIVERSITY OF BRITISH COLUMBIA

CALIFORNIA INSTITUTE OF TECHNOLOGY

UNIVERSITY OF CALIFORNIA

MONTANA STATE UNIVERSITY

UNIVERSITY OF NEVADA, RENO

NEW MEXICO STATE UNIVERSITY

OREGON STATE UNIVERSITY
UNIVERSITY OF OREGON

UNIVERSITY OF SOUTHERN CALIFORNIA

STANFORD UNIVERSITY

UNIVERSITY OF HAWAII

UNIVERSITY OF TOKYO

UNIVERSITY OF UTAH

WASHINGTON STATE UNIVERSITY

UNIVERSITY OF WASHINGTON 


\section{Pacific Journal of Mathematics}

\section{Vol. 103, No. $1 \quad$ March, 1982}

Abdul Aziz, On the zeros of composite polynomials ..................

Salomon Benzaquen and Enrique M. Cabaña, The expected measure of the level sets of a regular stationary Gaussian process $\ldots \ldots \ldots \ldots \ldots$

Claudio D’Antoni, Roberto Longo and László Zsidó, A spectral mapping theorem for locally compact groups of operators $\ldots \ldots \ldots \ldots \ldots \ldots \ldots 17$

Ronald Dotzel, Semifree finite group actions on homotopy spheres ........ 25

Daniel H. Gottlieb, The Lefschetz number and Borsuk-Ulam theorems . . . . . 29

Shui-Hung Hou, On property $(Q)$ and other semicontinuity properties of

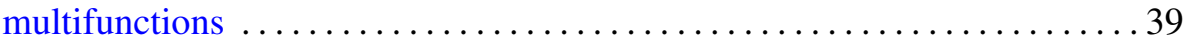

Kevin Mor McCrimmon, Compatible Peirce decompositions of Jordan triple systems

Mitsuru Nakai, Corona problem for Riemann surfaces of Parreau-Widom

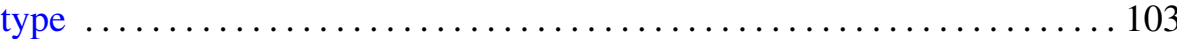

Jack Ray Porter and R. Grant Woods, Extensions of Hausdorff spaces . . . . 111

Milton Rosenberg, Quasi-isometric dilations of operator-valued measures

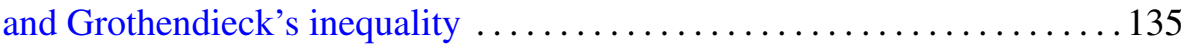

Joseph L. Taylor, A bigger Brauer group $\ldots \ldots \ldots \ldots \ldots \ldots \ldots \ldots \ldots \ldots$

Thomas Vogel, Symmetric unbounded liquid bridges . . . . . . . . . . . 205

Steve Wright, The splitting of operator algebras. II ............... 243 